\title{
Confident Privacy Decision-Making in loT Environments
}

\author{
HOSUB LEE, Samsung Research America, University of California, Irvine \\ ALFRED KOBSA, University of California, Irvine
}

\begin{abstract}
Researchers are building Internet of Things (IoT) systems that aim to raise users' privacy awareness, so that these users can make informed privacy decisions. However, there is a lack of empirical research on the practical implications of informed privacy decision-making in IoT. To gain deeper insights into this question, we conducted an online study $(N=488)$ of people's privacy decision-making as well as their levels of privacy awareness toward diverse IoT service scenarios. Statistical analysis on the collected data confirmed that people who are well aware of potential privacy risks in a scenario tend to make more conservative and confident privacy decisions. Machine learning (ML) experiments also revealed that individuals overall privacy awareness is the most important feature when predicting their privacy decisions. We verified that ML models trained on privacy decisions made with confidence can produce highly accurate privacy recommendations for users (area under the ROC curve (AUC) of 87\%). Based on these findings, we propose functional requirements for privacy-aware systems to facilitate well-informed privacy decision-making in IoT, which results in conservative and confident decisions that enjoy high consistency.
\end{abstract}

CCS Concepts: - Security and privacy $\rightarrow$ Human and societal aspects of security and privacy; • Human-centered computing $\rightarrow$ Empirical studies in ubiquitous and mobile computing; User studies; - Computing methodologies $\rightarrow$ Supervised learning by classification; Cluster analysis; Ensemble methods;

Additional Key Words and Phrases: IoT, privacy-aware system, privacy awareness, informed privacy decisionmaking, privacy decision support, decision confidence, random effects model, random forest

\section{ACM Reference format:}

Hosub Lee and Alfred Kobsa. 2019. Confident Privacy Decision-Making in IoT Environments. ACM Trans. Comput.-Hum. Interact. 27, 1, Article 6 (December 2019), 39 pages.

https://doi.org/10.1145/3364223

\section{INTRODUCTION}

Companies and organizations increasingly deploy intelligent Internet of Things (IoT) services that collect and analyze various types of available sensor data about the user. For instance, a company can operate an IoT service allowing employees to freely enter the building without badges by automatically verifying their identity based on face photos (captured by smart security cameras). ${ }^{1}$

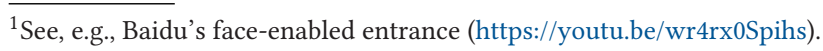

This work was done while H. Lee was a Ph.D. student at the University of California, Irvine.

This research has been supported by NSF grant SBE-1423629 and SES-1640527. The human subjects research described herein is covered under IRB protocol \#2014-1600 at the University of California, Irvine.

Authors' addresses: H. Lee, 18500 Von Karman Avenue, Suite 700, Irvine, CA 92612; email: hosub.lee1@samsung.com; A. Kobsa, 6091 Donald Bren Hall, Irvine, CA 92697-3440; email: kobsa@uci.edu.

Permission to make digital or hard copies of all or part of this work for personal or classroom use is granted without fee provided that copies are not made or distributed for profit or commercial advantage and that copies bear this notice and the full citation on the first page. Copyrights for components of this work owned by others than ACM must be honored. Abstracting with credit is permitted. To copy otherwise, or republish, to post on servers or to redistribute to lists, requires prior specific permission and/or a fee. Request permissions from permissions@acm.org.

(c) 2019 Association for Computing Machinery.

1073-0516/2019/12-ART6 \$15.00

https://doi.org/10.1145/3364223

ACM Transactions on Computer-Human Interaction, Vol. 27, No. 1, Article 6. Publication date: December 2019. 
Such kinds of IoT services can make people's lives more convenient and efficient. At the same time, however, users may have privacy concerns about information that can be inferred from sensor data. Researchers therefore argued that it is necessary to provide IoT users with technical means of controlling their privacy $[22,49,57,65,74,80]$, such as a feature to let them decide whether or not to use the IoT service (coarse-grained control) or to opt-out of the collection of specific sensor data (fine-grained control). Such a fine-grained privacy control in IoT is being actively investigated $[50,56]$, but it has not yet been deployed in a real-world setting.

However, even when control over the use of IoT services is available, users need to fully understand both the utility benefits and privacy risks in using IoT services, evaluate the balance between them (i.e., perform a utility-privacy tradeoff), and make a final privacy decision based on that evaluation. Yet in reality, it is difficult for them to do so because they are provided with no or only limited information to properly assess potential privacy risks of each IoT service. This lack of privacy risk awareness has been identified in previous research as one of the causes of the privacy paradox, ${ }^{2}$ which can result in undesirable privacy violations in ubiquitous computing environments [16]. For instance, IoT service providers tend to focus on promoting their services (e.g., automatic attendance check) while not informing the users about the sensor data (e.g., face photos) they are collecting and analyzing to provide those services. Informing users in detail about sensor data collection and analysis is important since due to the rapid advancements in data mining and machine learning (ML), all kinds of data can be used to infer potentially sensitive personal information (e.g., sexual orientation [73]). It is also possible to aggregate multiple types of data from different sensors, so as to infer personal information that previously seemed impossible to figure out.

A meaningful approach to help users make better privacy decisions therefore seems to be make them aware of privacy implications of an IoT service, specifically the types of personal information that can be inferred on the basis of the collected sensor data (i.e., informed privacy decisionmaking) [12,22]. However, it is still unclear what consequences greater user awareness of inferable personal information will have on their privacy decisions in IoT environments. In addition, it is worth investigating whether people's informed privacy decisions form a better basis for predicting their future privacy decisions (i.e., privacy decision support). Previous researchers have shown that privacy decision support can mitigate users' cognitive burden, thereby allowing them to make their preferred decisions more easily [9, 42, 77, 79].

To gain insights into these questions, we first conducted an online survey on Amazon Mechanical Turk $(N=488)$ to collect people's privacy decision-making about diverse IoT service scenarios that they may encounter in their everyday lives. We created 180 realistic scenarios while varying underlying contextual factors of the IoT service, such as location, purpose, relationships between collectible sensor data and inferable personal information, and data privacy policies (e.g., how data will be protected, retained, and shared). Each participant was successively presented with 15 randomly chosen scenarios and asked whether or not he or she would use the given IoT service (privacy decision). Along with each privacy decision, participants were prompted for their self-reported level of awareness about the inference of personal information (privacy awareness), perceived balance between utility benefits and privacy risks (utility-privacy tradeoffs), and their level of confidence in their privacy decision (decision confidence). We also constructed each individual's personal privacy propensity, which is composed of privacy segment, overall privacy awareness, and privacy self-efficacy, from survey responses separately collected before and after the abovementioned survey procedures.

\footnotetext{
${ }^{2}$ The privacy paradox is the phenomenon that people's stated privacy preferences or intentions often seem inconsistent with their actual behaviors $[2,25,53]$.
} 
Next, we conducted statistical analysis to understand which factors influence people's privacy decision-making in IoT environments. We analyzed the survey data with the following two different aims: (1) to understand how people make privacy decisions based upon their privacy awareness and other factors embedded in the scenarios, and (2) to understand how people have different levels of confidence in making such decisions. Regarding statistical models for analyzing people's privacy decisions (binary response) and their decision confidence (ordinal response), we used a generalized linear mixed model (GLMM) and cumulative link mixed model (CLMM), respectively. Through GLMM analysis, (1) we confirmed the fact that people who have a higher level of privacy awareness are more likely to make conservative decisions compared to those who do not. Through CLMM analysis, (2) we found statistical support for the fact that the level of people's privacy awareness is positively associated with their decision confidence, regardless of the type of decisions made. We therefore argue that guiding people to make conservative and confident decisions via the enhancement of privacy awareness is important. Conservative privacy decisions may imply people's willingness to minimize potential privacy risks by opting out of the service, thereby lowering the possibility of privacy violations. Confident privacy decision-making is also desirable since it may be the case that people are then more likely to make their IoT decisions reliably and consistently.

Lastly, we performed ML experiments with a Random Forest (RF) model, to check the feasibility of privacy decision support in IoT. We specifically aimed to investigate whether the level of users' confidence in privacy decisions impacts the predictive performance of the ML model. The experimental results indeed indicate that models exclusively trained on confident privacy decisions show superior accuracy in predicting people's decisions compared to models based on unconfident decisions (AUC of $87 \%$ vs. $67 \%$; statistically significant). Thus, we conclude that collecting confident privacy decisions is crucial for realizing privacy decision support that can give users meaningful privacy recommendations.

Based on the abovementioned findings, we propose functional requirements for privacy-aware systems (PAS) in IoT environments. PAS should be able to maximize users' awareness of potential privacy risks posed by IoT services (e.g., undesired inferences of personal information), thereby not only helping them make conservative decisions on their own but also allowing the PAS to accumulate confident decision samples, which can be used to later give users accurate privacy recommendations. We articulate specifically how PAS can better assist users in grasping privacy implications of using the IoT services, leading to their better-informed privacy decision-making.

In summary, our work makes the following contributions to the field of ubiquitous computing, with the ultimate aim of preserving user privacy in IoT:

-We propose methodologies to model each individual's personal privacy propensity, consisting of privacy segment, overall privacy awareness, and privacy self-efficacy, based on collected survey data regarding people's privacy decision-making behaviors toward IoT services.

- We extract several context- and user-specific privacy decision factors (including personal privacy propensity) that are significantly associated with the type and confidence of decisions made in IoT, and confirm that a higher level of privacy awareness is related to the likelihood of privacy decisions being made more conservatively and confidently.

- We demonstrate the feasibility of predicting people's privacy decisions via an ML method (AUC of up to 87\%) and verify that confident decision samples yield more accurate ML models for privacy decision support in IoT.

-We propose functional requirements for PAS to maximize people's privacy awareness in IoT, thereby nudging them to make more informed privacy decisions on increasingly diverse IoT services. 


\section{RELATED WORK}

In this section, we describe research efforts articulating the importance of privacy awareness in making informed privacy decisions, leading to minimized privacy risks. Next, we enumerate a set of different systems/methodologies that maximize people's privacy awareness in ubiquitous computing environments, including IoT. Lastly, we summarize the previous research works aimed at understanding and predicting people's privacy decisions in IoT environments, and explain how our work is different from others.

\subsection{Privacy Awareness on Privacy Decision-Making}

Privacy awareness is considered one of the key factors determining the type and quality of people's privacy decisions since it allows them to form more accurate mental models of the current situation $[3,55]$. Privacy awareness is the level of user perception of possible privacy risks from interacting with an application or service that can gather personal user data. For example, in online social network services (e.g., Facebook) users post photos and share them with their friends. However, some users are not aware of the fact that if they did not configure privacy settings properly, their photos might be shown to people they do not know. Without a full understanding of this kind of privacy risk, the user may make uninformed privacy decisions.

2.1.1 Online Privacy. Pötzsch stated that the reinforcement of privacy awareness is one possible solution for remedying the negative outcomes of uninformed privacy decisions [58], namely the privacy paradox. The author not only defined the attributes of privacy awareness in e-commerce and web environments but also argued for the necessity of tools, which enhance users' privacy awareness (e.g., an easily-understandable written privacy policy), thereby minimizing the inconsistency between privacy decisions and behaviors. The author also proposed several requirements for realizing such tools. In the same vein, Bergmann performed an online survey study to empirically show how the user's privacy awareness can be changed according to the user interface (UI) presenting privacy-related information on the web [8]. The author quantified users' levels of understanding of possible consequences of disclosing personal data online by showing them two different versions of data entry UIs: (1) a conventional login form collecting user ID and password and (2) a privacy-enhanced login form with clickable links for informing users about the detailed privacy policy (e.g., how the entered data will be protected by the server). Statistical analysis on the survey responses indicated that users who interacted with the privacy-enhanced form indeed have higher privacy awareness than the users of the conventional form.

2.1.2 Privacy in UbiComp. Könings et al. proposed a theoretical framework explaining how the user's privacy awareness can be modeled and enhanced in ubiquitous computing environments [31]. Using a graph-based model, the authors proposed a methodology to represent the data flow and information usage of a specific application (e.g., heart-rate monitoring) and to inform the user about potential privacy violations during its usage. Hong also stressed the importance of increasing privacy awareness to safeguard user privacy in a ubiquitously connected world [22].

\subsection{Enhancement of Privacy Awareness}

Due to the importance of privacy awareness in making informed decisions, many researchers have designed, developed, and tested computer systems that aim to maximize people's privacy awareness in ubiquitous computing environments.

2.2.1 Privacy-Aware System in UbiComp. Langheinrich proposed a PAS whose aim was not only to allow service providers to announce the details of how sensor data is collected but also to provide users with the technical means of managing how their personal information is inferred and 
stored by the service [33]. In order to achieve this goal, the author designed a networked system that enables the user to communicate with nearby services by exchanging machine-readable P3P privacy policies. ${ }^{3}$ Winkler et al. proposed a location-based PAS targeted at video surveillance systems [75]. The authors built trustworthy surveillance cameras that are capable of interacting with users through their smartphones, to inform them how their sensitive personal information (e.g., identity) is being handled by the cameras.

2.2.2 Privacy-Aware System in IoT. More recently, researchers are embedding privacy awareness in real-world IoT environments. Mehrotra et al. are developing a privacy-aware IoT framework named TIPPERS and deploying it in a university building $[50,56]$. TIPPERS is designed to allow its users to specify their preferred privacy settings (e.g., opt-out of specific data collection) and then systematically enforces that the IoT apps to follow these user preferences. To augment users' privacy awareness in IoT environments such as TIPPERS, Lee et al. prototyped a web-based PAS that broadcasts the visualized data privacy policies of IoT services via Bluetooth beacon technology [36]. Similarly, Das et al. proposed a PAS that allows the users to easily discover the privacy properties of IoT services using a smartphone app called IoT Assistants (IoTA) [14, 15, 72]. IoTA users can either configure privacy settings by themselves or get personalized recommendations based on their historical behaviors (privacy decision support).

\subsection{Modeling and Prediction of Privacy Decisions in loT}

As discussed above, there are a lot of research efforts in designing and developing systems with the aim of increasing people's privacy awareness in IoT environments. However, we find no empirical research that not only measures users' privacy awareness but also investigates the effects of the measured privacy awareness on self-reported or automated privacy decision-making in IoT. Instead, most of the previous works have focused on contextual factors (e.g., service ownership, location) that constitute a current situation, rather than each user's internal characteristics such as privacy awareness.

2.3.1 Contextualized Privacy Decisions. Lee and Kobsa collected end users' privacy attitudes and decisions toward hypothetical IoT service scenarios through both an online survey $(N=200)$ [37] and a field experiment $(N=172)$ [38]. The authors performed an unsupervised $K$-modes cluster analysis on the collected dataset and confirmed the fact that contextual parameters, such as the identity of an agent collecting sensor data (parameter who) and the type of personal information inferred from the collected sensor data (parameter what), significantly impact users' privacy decisions. They also trained a decision tree ML model that predicts users' binary privacy decisions based on their past decision-making behaviors, with an accuracy of $77 \%$ [38].

Using the dataset collected in [37], Bahirat et al. conducted a statistical analysis (linear mixed model; LMM) in order to determine underlying situational factors influencing users' privacy decisions toward the presented IoT scenarios [4]. Regarding this, the authors in $[4,37,38]$ reached the same conclusions, namely that parameters who and what have significant impacts on user-stated privacy decisions. Through the combination of $K$-means clustering and decision tree learning, Bahirat et al. also showed that it is possible to predict the users' future privacy decisions with an accuracy as high as $82 \%$.

Researchers have continued to study people's privacy decision-making in IoT. Emami-Naeini et al. conducted an online survey study in an experimental setting similar to that used by [37] but with larger sample populations $(N=1,007)$ [51]. The authors found that privacy decisions not only vary from person to person but are also highly context-dependent. According to their

$\overline{{ }^{3} \text { https://www.w3.org/TR/P3P/. }}$ 
GLMM analysis on the survey responses, the physical location where sensor data collection occurs, user-perceived benefit, and the type of collectible sensor data, significantly impact users' privacy decisions. To validate the feasibility of predicting privacy decisions, Emami-Naeini et al. trained logistic regression-based AdaBoost classifiers capable of predicting users' decisions of whether to accept or reject the IoT service. The average accuracy of their predictions was as high as $86 \%$.

2.3.2 Research Implication. All of the works mentioned above used similar methodologies in collecting and analyzing people's privacy decision behaviors in IoT, in that they presented hypothetical scenarios to online survey participants in which postulated predictor variables (contextual factors) are systematically varied. Researchers also adopted ML techniques to predict optimal privacy decisions for IoT users.

As discussed before, however, there is a lack of research to understand how people's privacy awareness impacts the type and quality of decisions they make. In this study, we therefore primarily investigate the role of privacy awareness in privacy decision-making in IoT. To the best of our knowledge, this is the first attempt to take privacy awareness into account when modeling and predicting privacy decisions in IoT.

\section{IOT PRIVACY DATASET}

In this section, we describe how we designed and performed an online survey asking the user's privacy attitudes and decisions about hypothetical IoT services. We also present our approach to characterize each user in three privacy-related dimensions such as privacy segment, overall privacy awareness, and privacy self-efficacy. We then explain how we prepared a dataset for performing statistical analysis and ML experiments.

\subsection{Data Collection}

3.1.1 IoT Service Scenario Generation. To gather users' opinions and reactions about their privacy in IoT, we need to present them with real IoT applications/services or hypothetical scenarios. Due to the lack of operational IoT environments, researchers choose the latter approach. One method involves generating IoT scenarios based on random permutations of contextual parameter values (e.g., who, what, when, where) [37, 51]. We extracted factors that construct IoT scenarios by referring to the IoT use cases collaboratively created by the IoT community [13, 54]. The factors include the service location (3 values), service purpose (4 values), and personal information inferable from the raw sensor data (12 values). We then created 15 representative base scenarios using certain meaningful combinations of these factor values (see Table 1). All participants responded to the same set of base scenarios, which cover all combinations of select factor levels. This subsampling of a full factorial design does not violate the applicability conditions for linear regression we will perform. We further randomized the sequence of the presented scenarios to avoid any order effects.

For each base scenario, we created a list of all possible and some impossible inferences of personal information from the collected sensor data. Regarding this, we first defined 34 possible and 22 impossible inferences based on the literature. Then, we convened a panel of three outside experts in the field of ML, computer vision, and ubiquitous computing who decided individually if each inference (if-then rule) is currently possible or not. For each inference rule, the type of sensor data and personal information are antecedent and consequent, respectively. No additional information (e.g., size and quality of sensor data) was given. We instructed them to make intuitive decisions based on their knowledge and experience, rather than referring to reference materials. Inter-rater reliability (Fleiss's kappa) was 0.57 at the significance level 0.05 . If there was no unison agreement between raters, a majority rule was applied. Note that the kappa of 0.57 is considered as a 
Table 1. Contextual Factors of Base Scenarios

\begin{tabular}{lccccc}
\hline ID & Location & Purpose & Core Inference $^{a}$ & \# Possible Inferences & Reference \\
\hline S01 & Private & Health & Vital $\Rightarrow$ Nothing & 1 & N/A \\
S02 & Private & Saving & Electricity Usage $\Rightarrow$ Energy Consumption Pattern & 4 & {$[40]$} \\
S03 & Private & Convenience & Photo $\Rightarrow$ Identity & 4 & {$[69]$} \\
S04 & Private & Convenience & Voice $\Rightarrow$ Device Control Intention & 4 & {$[18,27]$} \\
S05 & Private & Saving & Motion $\Rightarrow$ Presence & 3 & {$[19,76]$} \\
S06 & Private & Safety & Voice $\Rightarrow$ Identity & 3 & {$[60,71]$} \\
S07 & Private & Safety & OBD $\Rightarrow$ Nothing & 1 & N/A \\
S08 & Private & Safety & Photo $\Rightarrow$ Emotion & 4 & {$[5,32]$} \\
S09 & Work & Convenience & Photo $\Rightarrow$ Identity & 5 & {$[69]$} \\
S10 & Work & Convenience & Device ID $\Rightarrow$ User Location & 5 & {$[44,45]$} \\
S11 & Work & Health & Video $\Rightarrow$ Physical Activity & 4 & {$[52,61]$} \\
S12 & Work & Convenience & Video $\Rightarrow$ Presence & 5 & {$[39,43,68]$} \\
S13 & Public & Convenience & Photo $\Rightarrow$ Identity & 5 & {$[69]$} \\
S14 & Public & Convenience & Device ID $\Rightarrow$ User Location & 2 & {$[44,45]$} \\
S15 & Public & Safety & Video $\Rightarrow$ Identity & 5 & {$[39,43,68]$} \\
\hline
\end{tabular}

${ }^{a}$ Sensor Data $\Rightarrow$ Personal Information.

${ }^{b}$ On-board diagnostics data (e.g., RPM, speed, pedal position) of the connected car.

moderate agreement level [66]. We suspect that the lack of background information regarding the given inference was the main cause of the low consensus level among experts. Their different levels of awareness of the latest research would be one possible reason too. We will discuss this issue in a later section.

We then divided the possible inferences into a core inference and the remaining non-core inferences based on whether the inference is articulated in the scenario. For the base scenario S02, ${ }^{4}$ for instance, Electricity Usage $\Rightarrow$ Energy Consumption Pattern would be regarded as a core inference since the scenario explicitly describes this inference. The inference Electricity Usage $\Rightarrow$ Number of Household Members is also technically possible [78], but this is a non-core inference because it is not used to construct S02. On the other hand, Electricity Usage $\Rightarrow$ Emotion is currently an impossible inference to date. By considering both core and non-core inferences, we were able to determine the number of all possible inferences for each scenario. Appendix A.1 lists the textual descriptions and factor values of all base scenarios.

Lastly, we enhanced the 15 base scenarios by data privacy policies. We generated 12 different data privacy policies by systematically combining the factors such as data protection (2 values), retention (3 values), and sharing ( 2 values), and randomly assigned a data privacy policy to each base scenario. Therefore, different survey participants might see different data privacy policies for the same base scenario. Since we utilized equal probability to determine each factor level for generating the policy, we do not expect any validity issues regarding our regression analysis. Merging these additional factors with the base scenarios yielded a total of 180 IoT scenarios.

3.1.2 Survey Design. Our online survey will be conducted in the following sequence: (1) verifying respondents' comprehension of IoT and example services (IoT comprehension), (2) determination of each user's privacy segment (privacy segmentation), (3) modeling of privacy decisionmaking behaviors (privacy decision modeling), (4) verifying the attentiveness in taking the survey

\footnotetext{
${ }^{4}$ At your home, your smart electricity meter collects your electricity usage to infer your energy consumption patterns, thereby suggesting energy saving methods, for your savings.
} 


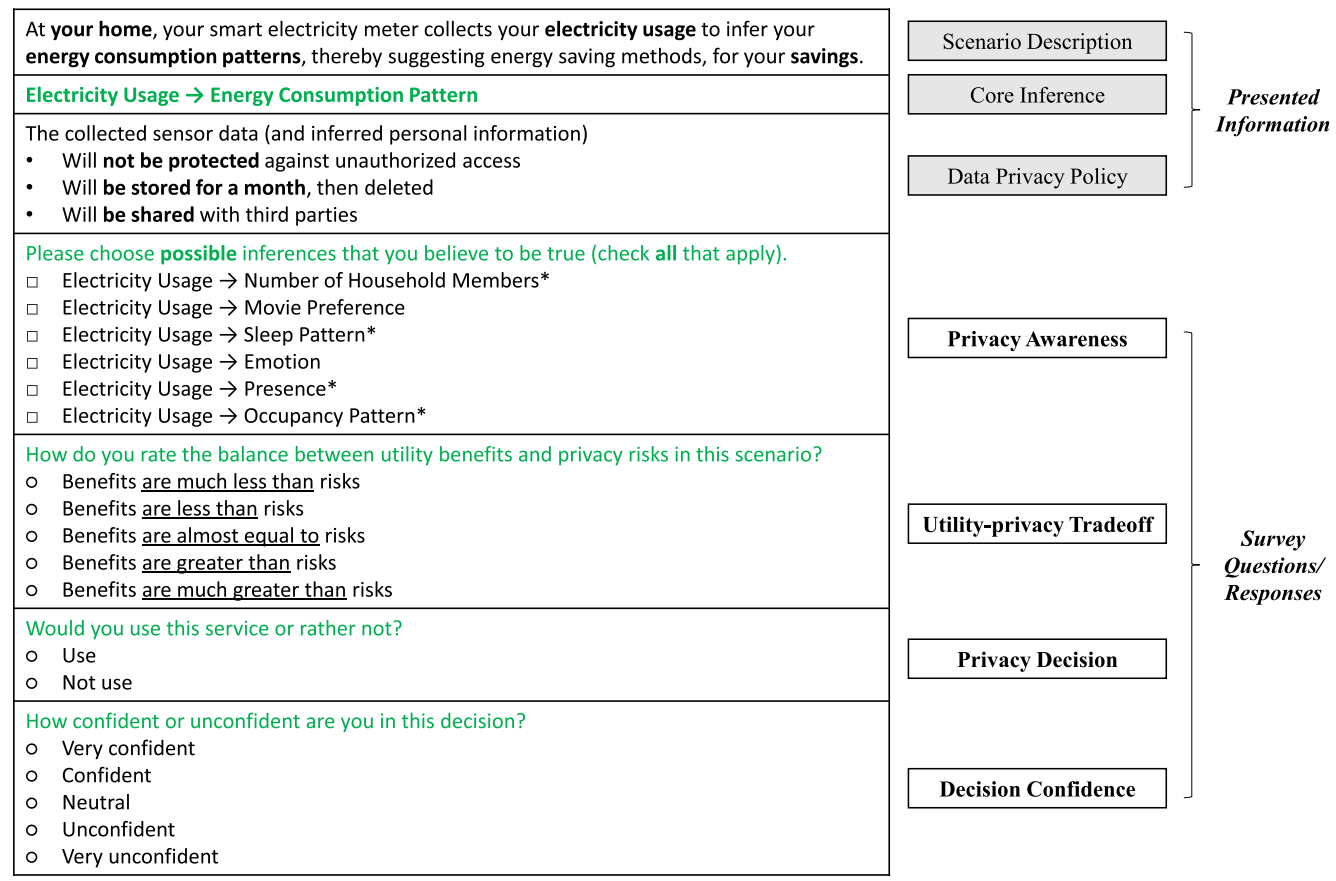

Fig. 1. Screenshot of main survey (Privacy decision modeling). Note: *denotes correct possible inference (not shown to participants).

(attention check), (5) privacy self-efficacy measurement, and (6) collection of demographic information of the survey population.

Step 1 To begin with, we presented survey participants with a brief definition of IoT, and some examples of real-world application (e.g., smart meter), in order to let them better understand the IoT context and then situate themselves in hypothetical but realistic scenarios we created. Participants are advised to read the information carefully and then asked three multiple-choice questions. We excluded participants from our data analysis who entered a wrong answer more than twice.

Step 2 To determine each participant's privacy segment, we created three hypothetical IoT services and a questionnaire similar to those in Lee and Kobsa [38]. We first generated service descriptions with varying underlying contextual information. Then we asked participants to answer five questions about their reaction attitudes toward the presented services.

Step 3 We then gathered people's decisions of whether or not to use the IoT service, along with their pre/post-decisional factors, namely privacy awareness, utility-privacy tradeoff, and decision confidence. A screenshot of a sample scenario (derived from base scenario S02, see Table 1) with all questions and answer options is depicted in Figure 1. We sequentially presented 15 random IoT scenarios from the available 180 scenarios. Each scenario is composed of the textual service description, the core inference of personal information as a form of if-then rule, and its data privacy policy. All contextual factors defining the scenario (see Section 3.1.1) are boldfaced to enable the participant to better understand it. To gauge the privacy awareness of participants, we asked them to mark additionally 
possible inferences of personal information in the given scenario. We presented all the pre-defined possible and impossible inferences as answer options. Next, we measured participants' utility-privacy tradeoff using a 5-pt Likert scale (e.g., 5 indicates that benefits are much less than risks). Participants make privacy decisions about whether or not to use the presented IoT scenario. Lastly, we inquired about their level of self-confidence regarding the decisions they made in the previous step on a 5-pt Likert scale (e.g., 5 indicates privacy decisions with high confidence).

Step 4 We aimed to check whether survey participants are paying enough attention to survey content. To that end, we informed them of the estimated remaining time and asked them on the next page to enter this value. We discarded participants who answered incorrectly.

Step 5 We measured privacy self-efficacy using a scale developed by LaRose and Rifon [35]. This scale consists of 10 items that gauge participants' familiarity and confidence regarding practices for safeguarding online privacy (e.g., I know how to change the privacy settings of my browser), on a 5-pt Likert scale.

Step 6 Lastly, we asked participants about their age, gender, length of residence in the US, education level, and annual household income, to understand the survey population and identify additional factors that potentially impact people's privacy decision-making in IoT. Also, we allowed participants to choose Prefer not to disclose (n/a: no answer) if they wanted.

Regarding Step 2 and 3, we actually performed a special kind of vignette study to collect our survey data. This is because we presented survey participants with scenario descriptions generated from the systematic combinations of predictor variables [62, 63]. This approach had been used in prior works studying people's privacy perceptions [47, 48].

We summarize the survey responses collectible through the abovementioned procedures in Table 2. We implemented and deployed our survey on SurveyMonkey. ${ }^{5}$

3.1.3 Survey Administration. We recruited 507 survey participants on Amazon Mechanical Turk (MTurk) in late March 2018. To assure the quality of survey responses, we restricted participants to adults who live in the US, are proficient in English, and have a high reputation on MTurk (above 97\% task approval rate and above 5,000 approved tasks). The recruited participants are provided with a link to our survey deployed at SurveyMonkey. All of them received \$2 as compensation if they completed the survey. ${ }^{6}$ As discussed before, we discarded participants if they failed to pass IoT comprehension or the attention-check question. We also eliminated some participants who finished the survey too rapidly (in less than 5 minutes, compared to the average response time of about 15 minutes). Thereby, we secured 488 qualified survey participants. Table 3 displays their demographics. We obtained approval for this study from IRB at our institution. A recruitment script and consent form can be found in Appendix A.2.

\subsection{Privacy Propensity Modeling}

Based on the collected survey responses, we extracted additional factors that represent people's personal privacy propensity, namely privacy segment, overall privacy awareness, and privacy selfefficacy.

3.2.1 Privacy Segment. Privacy segmentation, which groups users into specific segments according to the similarity of their privacy perception and behavior, can yield insights for

\footnotetext{
${ }^{5}$ https://www.surveymonkey.com/.

${ }^{6}$ We considered both the US federal minimum wage ( $\$ 7.25 /$ hour) and the average survey completion time ascertained in pilot testing (15 minutes; $N=6$ ). This yields a base rate of $\$ 1.8$, rounded up to $\$ 2$.
} 
Table 2. Summary of Survey Responses

\begin{tabular}{|c|c|c|c|}
\hline Purpose & Response & Description & Data Type \\
\hline $\begin{array}{c}\text { IoT } \\
\text { Comprehension }\end{array}$ & $\begin{array}{l}\text { Understanding } \\
\text { Level }\end{array}$ & $\begin{array}{l}\text { Ratio of correct answers to all IoT-related } \\
\text { questions }\end{array}$ & Numerical $(0.0 \sim 1.0)$ \\
\hline \multirow{5}{*}{$\begin{array}{c}\text { Privacy } \\
\text { Segmentation }\end{array}$} & $\begin{array}{c}\text { Notification } \\
\text { Preference }\end{array}$ & $\begin{array}{l}\text { Intention to be notified about data collection } \\
\text { being performed by IoT service }\end{array}$ & Categorical (4-class) \\
\hline & $\begin{array}{l}\text { Permission } \\
\text { Preference }\end{array}$ & $\begin{array}{l}\text { Intention to allow or reject data collection } \\
\text { being performed by IoT service }\end{array}$ & Categorical (4-clas \\
\hline & $\begin{array}{c}\text { Perceived } \\
\text { Comfort }\end{array}$ & $\begin{array}{l}\text { Perceived level of comfort about data collection } \\
\text { being performed by IoT service }\end{array}$ & Ordinal (7pt-scale) \\
\hline & Perceived Risk & $\begin{array}{l}\text { Perceived level of risk about data collection } \\
\text { being performed by IoT service }\end{array}$ & Ordinal (7pt-scale) \\
\hline & $\begin{array}{c}\text { Perceived } \\
\text { Appropriateness }\end{array}$ & $\begin{array}{l}\text { Perceived level of appropriateness about data } \\
\text { collection being performed by IoT service }\end{array}$ & Ordinal (7pt-scale) \\
\hline \multirow{4}{*}{$\begin{array}{l}\text { Privacy Decision } \\
\text { Modeling }\end{array}$} & $\begin{array}{c}\text { Privacy } \\
\text { Awareness }\end{array}$ & $\begin{array}{l}\text { Ratio of correct answers to all questions about } \\
\text { possible inferences in given IoT service }\end{array}$ & Numerical $(0.0 \sim 1.0)$ \\
\hline & $\begin{array}{l}\text { Utility-Privacy } \\
\text { Tradeoff }\end{array}$ & $\begin{array}{l}\text { Perceived balance between utility benefits and } \\
\text { privacy risks in given IoT service }\end{array}$ & Ordinal (5pt-scale) \\
\hline & Privacy Decision & Intention to use or not to use given IoT service & Categorical (binary) \\
\hline & $\begin{array}{l}\text { Decision } \\
\text { Confidence }\end{array}$ & $\begin{array}{l}\text { Perceived level of confidence in making a } \\
\text { privacy decision for given IoT service }\end{array}$ & Ordinal (5pt-scale) \\
\hline Attention Check & User Attention & $\begin{array}{l}\text { Indication of whether the participant is paying } \\
\text { attention while participating }\end{array}$ & Categorical (binary) \\
\hline $\begin{array}{c}\text { Privacy } \\
\text { Self-efficacy } \\
\text { Measurement }\end{array}$ & Agreement Level & $\begin{array}{l}\text { Level of agreement with presented statements } \\
\text { on online privacy self-management }\end{array}$ & Ordinal (5pt-scale) \\
\hline \multirow{5}{*}{ Demographic } & Age & Age group & Categorical (6-class) \\
\hline & Gender & Gender identity & Categorical (3-class) \\
\hline & Residence & Length of residence in the US & Categorical (5-class) \\
\hline & Education & Highest level of education & Categorical (8-class) \\
\hline & Income & Annual household income level & Categorical (7-class) \\
\hline
\end{tabular}

Note: Categorical/ordinal values of the survey response are as follows.

- Notification: (1) Notify, always, (2) Notify, just this time, (3) Do not notify, just this time, (4) Do not notify, always

- Permission: (1) Allow, always, (2) Allow, just this time, (3) Reject, just this time, (4) Reject, always

- Comfort: (1) Very uncomfortable, ..., (7) Very comfortable

- Risk: (1) Very risky, ..., (7) Very safe

- Appropriateness: (1) Very inappropriate, ..., (7) Very appropriate

- Utility-privacy Tradeoff: (1) Benefits $\gg$ Risks, . . ., (5) Benefits « Risks

- Privacy Decision: (0) Not use, (1) Use

- Decision Confidence: (1) Very unconfident, ..., (5) Very confident

- User Attention: (0) No attention, (1) Attention

- Agreement Level: (1) Strongly disagree, ..., (5) Strongly agree.

understanding people's privacy decision-making in various circumstances [29, 34]. Privacy segment information is also proven to be particularly useful for predicting people's privacy decisions $[41,42]$. Since one of the ultimate aims of our study is helping IoT users with making accurate privacy recommendations, we determined to utilize privacy segmentation as one of the input features for predicting people's preferred privacy decisions. The most common approach for privacy segmentation is running an unsupervised clustering algorithm such as $K$-means on user-provided data (e.g., survey responses). This kind of algorithms is designed to assign each data point (i.e., user) into a single cluster, rather than a compound cluster. 
Table 3. Demographic Breakdown of Survey Population

\begin{tabular}{|c|c|c|c|c|c|c|c|c|c|}
\hline \multicolumn{2}{|c|}{ Age } & \multicolumn{2}{|c|}{ Gender } & \multicolumn{2}{|c|}{ US Residence (yr) } & \multicolumn{2}{|c|}{ Education } & \multicolumn{2}{|l|}{ Income } \\
\hline $18-24$ & $4.7 \%$ & Male & $48.6 \%$ & $0-4$ & $0.2 \%$ & $<$ High School & $0.8 \%$ & $<\$ 20,000$ & $10.9 \%$ \\
\hline $25-34$ & $36.9 \%$ & Female & $51.2 \%$ & $5-10$ & $0.8 \%$ & High School & $25.2 \%$ & $\$ 20,000-\$ 34,999$ & $19.3 \%$ \\
\hline $35-44$ & $28.9 \%$ & $\mathrm{n} / \mathrm{a}$ & $0.2 \%$ & $11-20$ & $1.2 \%$ & Associate & $21.1 \%$ & $\$ 35,000-\$ 49,999$ & $18.6 \%$ \\
\hline $45-54$ & $14.8 \%$ & & & $>20$ & $96.9 \%$ & Bachelor & $41.2 \%$ & $\$ 50,000-\$ 74,999$ & $24.0 \%$ \\
\hline $55-64$ & $11.3 \%$ & & & $\mathrm{n} / \mathrm{a}$ & $0.8 \%$ & Master & $8.0 \%$ & $\$ 75,000-\$ 99,999$ & $14.3 \%$ \\
\hline \multirow[t]{3}{*}{$>65$} & $3.5 \%$ & & & & & Professional & $1.4 \%$ & $>\$ 100,000$ & $10.7 \%$ \\
\hline & & & & & & Doctorate & $1.4 \%$ & $\mathrm{n} / \mathrm{a}$ & $2.3 \%$ \\
\hline & & & & & & $\mathrm{n} / \mathrm{a}$ & $0.8 \%$ & & \\
\hline
\end{tabular}

Note: Our study participants were mostly in their 20s and 30s. Most of them have lived in the US for more than 20 years, have bachelor degrees, and have annual incomes of more than $\$ 50,000$.

Table 4. Cluster Centroids for Sample Scenario

\begin{tabular}{cccccc}
\hline Privacy Segment & Notification & Permission & Comfort & Risk & Appropriateness \\
\hline Somewhat Insensitive & $\begin{array}{c}\text { Allow, } \\
\text { just this time }\end{array}$ & $\begin{array}{c}\text { Somewhat } \\
\text { comfortable }\end{array}$ & Neutral & $\begin{array}{c}\text { Somewhat } \\
\text { appropriate }\end{array}$ & $\begin{array}{c}\text { Notify, } \\
\text { always }\end{array}$ \\
\hline Somewhat Sensitive & $\begin{array}{c}\text { Reject, } \\
\text { just this time }\end{array}$ & $\begin{array}{c}\text { Somewhat } \\
\text { uncomfortable }\end{array}$ & $\begin{array}{c}\text { Somewhat } \\
\text { risky }\end{array}$ & $\begin{array}{c}\text { Somewhat } \\
\text { inappropriate }\end{array}$ & $\begin{array}{c}\text { Notify, } \\
\text { always }\end{array}$ \\
\hline Very Sensitive & $\begin{array}{c}\text { Reject, } \\
\text { always }\end{array}$ & $\begin{array}{c}\text { Very } \\
\text { uncomfortable }\end{array}$ & $\begin{array}{c}\text { Very } \\
\text { risky }\end{array}$ & $\begin{array}{c}\text { Very } \\
\text { inappropriate }\end{array}$ & $\begin{array}{c}\text { Notify, always } \\
\text { alwatian }\end{array}$ \\
\hline
\end{tabular}

Note: Notification, Permission, Comfort, Risk, Appropriateness columns denote the representative values for each resulting user cluster (privacy segment).

We performed privacy segmentation as follows. Based on [38], we first created three representative IoT scenarios and collected the reaction attitudes of our survey participants for each scenario (see Appendix A.3). Then we performed $K$-modes ${ }^{7}$ cluster analysis to segment users based on the commonality of their responses. To begin with, we determined the optimal number of clusters $(K)$ through the Elbow method; we found out that for a specific scenario, the largest decrease in clustering errors occurred when we increased $K$ from 2 to 3 . Therefore, we chose 3 as a suitable number of clusters for this scenario. We repeated this procedure against the remaining two scenarios and drew the same conclusion $(K=3)$. Next, we ran the $K$-modes clustering algorithm ${ }^{8}$ on our data in order to determine each participant's cluster membership (privacy segment). Table 4 presents the cluster centroids learned from the user responses to the specific scenario. These centroids are formulated with the representative reaction attitude values for the corresponding cluster. As it can be seen, each cluster is quite distinct, primarily in the perceived level of comfort, risk, and appropriateness: each centroid has a unique combination of these reaction values. Therefore, we marked the clusters based on these three reactions (see Privacy Segment column in Table 4). We also statistically validated the distinctiveness of the clustering results by conducting Welch's $t$-tests. ${ }^{9}$ The tests confirm that the difference in the means of each reaction value between each pair of the resulting segments is statistically significant $(p<0.025$, Bonferroni-corrected for two comparisons). Again, we repeated this procedure for the remaining scenarios, and came up with similar results.

\footnotetext{
${ }^{7}$ As a variant of $K$-means, $K$-modes clustering $[23,24]$ is designed to cluster categorical (or ordinal) values without data conversions.

${ }^{8}$ We used klaR package (https://cran.r-project.org/web/packages/klaR/), an R implementation of $K$-modes, for the task of privacy segmentation.

${ }^{9}$ The reason for using Welch's $t$-test is that all privacy segments have different variances in all reaction values.
} 
Table 5. Confirmatory Factor Analysis for Privacy Self-efficacy

\begin{tabular}{clcc}
\hline $\mathrm{ID}^{a}$ & \multicolumn{1}{c}{ Item (from LaRose and Rifon 2007) } & $\mathrm{FL}^{b}$ & $\mathrm{R}^{2}$ \\
\hline PSE1 & It's easy to figure out which sites you can trust on the Internet. & 0.68 & 0.47 \\
PSE2 & I am confident I know how to protect my credit card information online. & $\mathbf{0 . 8 4}$ & $\mathbf{0 . 7 1}$ \\
PSE3 & I know how to identify sites with secure servers. & $\mathbf{0 . 7 6}$ & $\mathbf{0 . 5 7}$ \\
PSE4 & I know how to evaluate online privacy policies. & $\mathbf{0 . 7 6}$ & $\mathbf{0 . 5 8}$ \\
PSE5 & It's easy to set up dummy email account to shield my identity. & 0.60 & 0.37 \\
PSE6 & I know how to change the security settings of my browser to increase privacy. & $\mathbf{0 . 7 1}$ & $\mathbf{0 . 5 1}$ \\
PSE7 & I know how to use a virus-scanning program. & 0.63 & 0.40 \\
PSE8 & I am able to protect myself against the release of personal information. & $\mathbf{0 . 7 5}$ & $\mathbf{0 . 5 7}$ \\
PSE9 & I know how to block unwanted E-mails. & 0.64 & 0.40 \\
PSE10 & Overall, I am confident that I can protect my privacy online. & $\mathbf{0 . 8 5}$ & $\mathbf{0 . 7 2}$ \\
\hline
\end{tabular}

${ }^{a}$ Indicator.

${ }^{b}$ Factor loading.

Ideally, the results of privacy segmentation should be independent of the scenario used for clustering. However, we noticed that some participants (about 13\%) get clustered into three different privacy segments. It is possible that these participants reacted differently to each scenario. Accordingly, we marked their segments as undecided. Otherwise, we adopted the majority voting approach to determine an individual's privacy segment. As a result, $26 \%$ of participants were classified as somewhat privacy insensitive $(N=127), 17 \%$ as somewhat sensitive $(N=85)$, and $43 \%$ as very sensitive $(\mathrm{VS})(N=211)$.

3.2.2 Overall Privacy Awareness. As discussed, we measured the privacy awareness of the participants by asking them to choose additional inferences of personal information that they believed to be true. For each of the 15 presented scenarios, we calculated a participant's privacy awareness as the ratio of correct answers. We used a modified mean of the measured scores after excluding the highest and lowest score as a measure of overall privacy awareness of the participant (population means $0.72, S D=0.15)$.

3.2.3 Privacy Self-efficacy. Privacy self-efficacy is defined as people's self-confidence or belief of abilities in protecting their privacy by themselves. We adopted LaRose and Rifon's scale [35] for quantifying the level of privacy self-efficacy of our survey participants (see Table 5) and tested whether our survey data fit this hypothesized privacy self-efficacy measurement model via confirmatory factor analysis (CFA) [10]. To be specific, we fitted a CFA model ${ }^{10}$ on our survey responses toward all the presented indicators (i.e., full model). In doing this, we used a weighted least squares estimator (e.g., WLSMV) since it provides the optimal way for modeling categorical/ordinal data without an assumption of data normality. The full CFA model showed that 4 indicators should be dropped since they have unsatisfactory factor loadings $<0.7$.

Considering only the indicators with satisfactory factor loadings, we fitted a reduced CFA model with a reasonable convergent validity $(A V E=0.61)$. Based on the fitted reduced model $\left(\chi^{2} / d f=\right.$ $3.85, C F I=0.928, R M S E A=0.07$ ), we computed the factor score (standardized weighted average value of the latent variable based on factor loadings) for each participant. We then treated the computed factor score as the measurement of his/her privacy self-efficacy. The measured values of privacy self-efficacy range from -1.7 to $2.4(M=0.00, S D=0.79)$.

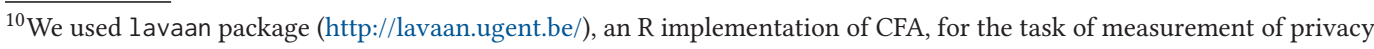
self-efficacy.
} 
Table 6. Dataset Summary

\begin{tabular}{|c|c|c|c|c|c|c|}
\hline Category & Attribute & Type & Range & GLMM & CLMM & $\mathrm{RF}$ \\
\hline $\mathrm{N} / \mathrm{A}$ & Subject ID & Cate. & 488-class & Random IV & Random IV & Excluded \\
\hline \multirow{7}{*}{$\begin{array}{l}\text { Contextual } \\
\text { Information }\end{array}$} & Location & Cate. & 3-class & Fixed IV & Fixed IV & Feature \\
\hline & Purpose & Cate. & 4-class & Fixed IV & Fixed IV & Feature \\
\hline & Core Inference & Cate. & 12-class & Fixed IV & Fixed IV & $\overline{\text { Feature }}$ \\
\hline & Data Protection & Cate. & 2-class & Fixed IV & Fixed IV & Feature \\
\hline & Data Retention & Cate. & 3-class & Fixed IV & Fixed IV & Feature \\
\hline & Data Sharing & Cate. & 2-class & Fixed IV & Fixed IV & Feature \\
\hline & \# Possible Inferences & Nume. & {$[0,1]$} & Fixed IV & Fixed IV & Feature \\
\hline \multirow{4}{*}{$\begin{array}{c}\text { Privacy } \\
\text { Decision-making } \\
\text { Behavior }\end{array}$} & Privacy Awareness & Nume. & {$[0,1]$} & Fixed IV & Fixed IV & Feature \\
\hline & Utility-privacy Tradeoff & Ordinal & 5-class & Fixed IV & Fixed IV & Excluded \\
\hline & Privacy Decision & Binary & 2-class & DV & Fixed IV & Label \\
\hline & Decision Confidence & Ordinal & 5-class & Excluded & DV & Excluded \\
\hline \multirow{3}{*}{$\begin{array}{l}\text { Personal Privacy } \\
\text { Propensity }\end{array}$} & Overall Privacy Awareness & Nume. & {$[0,1]$} & Fixed IV & Fixed IV & Feature \\
\hline & Privacy Segment & Cate. & 4-class & Fixed IV & Fixed IV & Feature \\
\hline & Privacy Self-efficacy & Nume. & {$[0,1]$} & Fixed IV & Fixed IV & Feature \\
\hline \multirow{5}{*}{$\begin{array}{l}\text { Demographic } \\
\text { Information }\end{array}$} & Age & Cate. & 6-class & Fixed IV & Fixed IV & Feature \\
\hline & Gender & Cate. & 3-class & Fixed IV & Fixed IV & Feature \\
\hline & Residence & Cate. & 5-class & Fixed IV & Fixed IV & Feature \\
\hline & Education & Cate. & 8-class & Fixed IV & Fixed IV & Feature \\
\hline & Income & Cate. & 7-class & Fixed IV & Fixed IV & Feature \\
\hline
\end{tabular}

\subsection{Dataset Summary}

Through the abovementioned procedures of online survey administration and privacy propensity modeling, we created a dataset with 19 attributes that characterize both the IoT-enabled spaces and users who interact with them (see Table 6). There exist 7 attributes with contextual information about IoT services, 4 attributes for users' privacy decisions and pre/post-decisional factors (privacy decision-making behavior), 3 attributes for indicating their privacy propensity, and 5 attributes for demographic information. A total of 15 attributes are categorical/ordinal and 4 numerical. As 488 survey participants individually responded to 15 scenarios, we came up with 7,320 user-scenario instances. There were 2,916 accept decisions (use the service) and 4,404 rejects.

The dataset is now fed into our statistical (GLMM and CLMM) and ML (RF) models to determine underlying factors that yield conservative and confident privacy decisions, and to investigate the effects of the decision confidence on the prediction of future decisions. Most of the attributes will be treated as independent variables (IVs) for statistical models or input features for ML models. A privacy decision attribute, in contrast, will be used as a dependent variable (DV) or a label to be predicted (see the last three columns in Table 6). Note that we excluded utility-privacy tradeoff from the input features for building RF models since it only constitutes a semi-final privacy decision. Regarding the numerical attributes, we performed min-max normalization in order to rescale the range of original values to $[0,1]$, thereby achieving better fitted predictive models.

\section{ANALYZING PRIVACY DECISION-MAKING IN IOT}

In this section, we explain how we statistically analyzed people's privacy decision-making in more detail. First, we not only identify factors that have a meaningful effect on binary privacy decisions in IoT environments but also interpret how these factors impact the type of decisions (e.g., 
permissive vs. conservative). In addition, we investigate which factors influence the user-perceived confidence about the decisions made (e.g., confident vs. unconfident), and how. Specifically, we focus on the degree of people's privacy awareness as one of the key predictor variables. We are therefore interested in the following research questions:

RQ1: If people have sufficient awareness of potentially inferable personal information when using an IoT service, will they make their privacy decisions more conservatively, or will they rather disclose more openly, or will there be no difference?

RQ2: If people have sufficient awareness of potentially inferable personal information when using an IoT service, will they make their privacy decisions more confidently or less confidently, or will there be no difference?

\subsection{Factors Impacting Privacy Decisions}

In order to model privacy decision factors in IoT, we adopted a GLMM regression [6] with a random intercept per participant. GLMM is an extension of the generalized linear model (GLM) in which the linear covariates contain both fixed and random effects. For the analysis of the grouped data, GLMM can model the differences between groups as a random effect. GLMM is suited for analyzing our survey data since each of our participants responded to multiple parallel scenarios (grouped by subject); it enables us to take the within-subject associations into account when interpreting the analysis results.

4.1.1 Problem Definition. Modeling privacy decision factors can be defined as follows. Consider a GLMM model with $p$ covariates for the probability of the dichotomous response $Y_{i j}$ of the subject $i(1, \ldots, N)$ for the item $j\left(1, \ldots, n_{i}\right)$ being equal to one:

$$
\operatorname{Logit}\left(P\left(Y_{i j}=1\right)\right)=x_{i j}^{T} \beta+v_{i},
$$

where $Y_{i j}$ is the binary privacy decision of the $i$ th subject toward the $j$ th scenario (1: use, 0 : not use); $x_{i j}$ is the $(p+1)$-dimensional vector of IVs (i.e., fixed effects); $\beta$ is the $(p+1)$-dimensinal vector of regression coefficients; $v_{i}$ is the random subject effects distributed iid-normal: $v_{i} \sim \mathcal{N}\left(0, \sigma_{v}^{2}\right)$; $N$ is the number of subjects (488); and $n_{i}$ is the number of scenarios shown to the $i$ th subject (15). We specified a logit link function since we assumed that the probability distribution of $Y_{i j}=1$ is binomial.

4.1.2 Model Selection. We performed model selection to find the best combination of privacy decision factors through a backward elimination approach [26]. Our model selection routine starts with fitting a model with the most complex structure possible given the specified combination of fixed effects and their interactions. It then performs backward stepwise selection to obtain the minimum adequate model based on likelihood ratio test (LRT). Interaction terms are tested first, and then removed to test each fixed effect. All fixed effects that are part of significant interaction terms are retained in the final model regardless of their significance level. The specified random effects are fixed. A maximum likelihood (ML) estimator is used for selecting privacy decision factors and then restricted maximum likelihood (REML) estimator is used for fitting the final reduced model.

Though the abovementioned procedures, we fitted the reduced GLMM model ${ }^{11}$ as shown below:

$$
\begin{aligned}
\operatorname{Logit}\left(P\left(Y_{i j}=1\right)\right)= & \beta_{0}+\beta_{1}\left(\text { location }_{i j}\right)+\beta_{2}\left(\text { infer }_{i j}\right)+\beta_{3}\left(\text { protect }_{i j}\right)+\beta_{4}\left(\text { share }_{i j}\right) \\
& +\beta_{5}\left(\text { aware }_{i j}\right)+\beta_{6}\left(\text { tradeoff }_{i j}\right)+\beta_{7}\left(\text { segment }_{i}\right)+\beta_{8}\left(\text { efficacy }_{i}\right)+v_{i},
\end{aligned}
$$

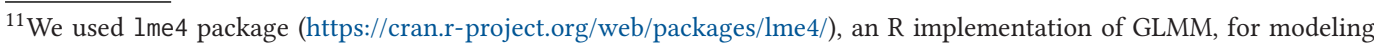
privacy decision factors.
} 
Table 7. Regression Results of GLMM Model

\begin{tabular}{|c|c|c|c|c|c|}
\hline Factor Impacting Privacy Decision & Coef $\beta$ & $\mathrm{SE}(\beta)$ & $\mathrm{z}$ & $p$ & OR $(95 \% \mathrm{CI})$ \\
\hline Intercept & 5.71 & 0.40 & 14.37 & $* * *$ & $301.16(138.25-656.04)$ \\
\hline location:Private & \multicolumn{5}{|c|}{ baseline } \\
\hline location:Work & 1.64 & 0.22 & 7.61 & $* * *$ & $5.13(3.37-7.82)$ \\
\hline location: Public & 1.40 & 0.21 & 6.53 & $* * *$ & $4.04(2.66-6.15)$ \\
\hline infer:DeviceID $\Rightarrow$ UserLocation & \multicolumn{5}{|c|}{ baseline } \\
\hline infer:Electricity $\Rightarrow$ EnergyConsumptionPattern & 2.89 & 0.28 & 10.35 & $* * *$ & $18.05(10.43-31.22)$ \\
\hline infer:Motion $\Rightarrow$ Presence & 2.65 & 0.28 & 9.57 & $* * *$ & $\mathbf{1 4 . 2 2}(8.26-24.51)$ \\
\hline infer: Video $\Rightarrow$ Presence & -0.08 & 0.21 & -0.39 & & $0.92(0.61-1.39)$ \\
\hline infer:Voice $\Rightarrow$ DeviceControlIntention & 2.26 & 0.28 & 8.08 & $* * *$ & $9.62(5.56-16.67)$ \\
\hline infer:Voice $\Rightarrow$ Identity & 1.87 & 0.27 & 6.80 & $* * *$ & $6.48(3.78-11.11)$ \\
\hline infer:Photo $\Rightarrow$ Identity & 0.94 & 0.17 & 5.60 & $* * *$ & $2.56(1.84-3.56)$ \\
\hline infer:Video $\Rightarrow$ Identity & 0.83 & 0.21 & 4.00 & $* * *$ & $2.29(1.52-3.43)$ \\
\hline infer:Video $\Rightarrow$ PhysicalActivity & -0.71 & 0.22 & -3.28 & $* *$ & $\mathbf{0 . 4 9}(0.32-0.75)$ \\
\hline infer:Photo $\Rightarrow$ Emotion & 0.12 & 0.31 & 0.40 & & $1.13(0.62-2.08)$ \\
\hline infer:OBD $\Rightarrow$ Nothing & 2.77 & 0.28 & 10.03 & $* * *$ & $15.88(9.25-27.27)$ \\
\hline infer:Vital $\Rightarrow$ Nothing & 2.36 & 0.27 & 8.61 & $* * *$ & $10.63(6.2-18.2)$ \\
\hline protect:Protected & \multicolumn{5}{|c|}{ baseline } \\
\hline protect: Unprotected & -0.14 & 0.08 & -1.67 & & $0.87(0.74-1.02)$ \\
\hline share:Unshared & \multicolumn{5}{|c|}{ baseline } \\
\hline share:Shared & -0.19 & 0.08 & -2.35 & * & $\mathbf{0 . 8 2}(0.7-0.97)$ \\
\hline aware & -0.81 & 0.21 & -3.93 & $* * *$ & $0.45(0.3-0.67)$ \\
\hline tradeoff & -2.06 & 0.06 & -34.86 & $* * *$ & $\mathbf{0 . 1 3}(0.11-0.14)$ \\
\hline segment:SomewhatInsensitive & \multicolumn{5}{|c|}{ baseline } \\
\hline segment: SomewhatSensitive & -0.45 & 0.24 & -1.91 & & $0.64(0.4-1.01)$ \\
\hline segment:VerySensitive & -1.51 & 0.20 & -7.62 & $* * *$ & $0.22(0.15-0.32)$ \\
\hline segment: Undecided & -0.19 & 0.26 & -0.72 & & $0.83(0.5-1.37)$ \\
\hline efficacy & 0.95 & 0.42 & 2.25 & $*$ & $2.59(1.13-5.93)$ \\
\hline
\end{tabular}

Note: $* p<0.05, * * p<0.01, * * * p<0.001$.

where $\left\{\right.$ location $_{i j}$, infer ${ }_{i j}$, protect $_{i j}$, share $\left.{ }_{i j}\right\}$ represent contextual information of the $j$ th scenario shown to the $i$ th subject, namely its service location, core inference, data protection and data sharing; $\left\{\right.$ aware $_{i j}$, tradeoff $f_{i j}$ \} is the measured privacy awareness and utility-privacy tradeoff ${ }^{12}$ of the $i$ th subject toward the $j$ th scenario (i.e., pre-decisional factors); $\left\{\right.$ segment $_{i}$, efficacy $\left.{ }_{i}\right\}$ are the determined privacy segment and self-efficacy of the $i$ th subject (i.e., personal privacy propensity); $\beta_{0}$ is an intercept; and $\beta_{p}$ is a regression coefficient of the $p$ th IV $(p=1, \ldots, 8) . Y_{i j}$ and $v_{i}$ are the same as denoted by Equation (1).

4.1.3 Interpretation. We present the regression results of the fitted GLMM model in Table 7. As can be seen, most factors are associated with the dependent privacy decision variable. However, it is also true that not all levels of the categorical factors are statistically significant. Below we interpret the results based on the odds ratio (OR) computed for each factor category with $p<0.05$ (marked out in bold).

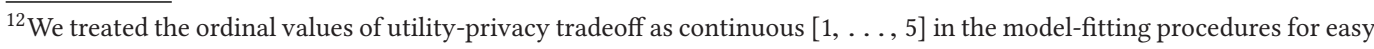
interpretation.
} 
Contextual information. Regarding contextual information, we confirmed that the current location, type of inferable personal information, and data sharing practice significantly impact people's privacy decisions. Since most of the interpretations are consistent with the literature, we then described them in Appendix A.4 to focus on the results related to the research question that we investigate.

Privacy awareness. Regression results showed that privacy awareness significantly impact people's privacy decision-making in IoT. We verified that people who are knowledgeable about possible inferences of personal information in IoT environments tend to be conservative when they make decisions about the use of services $(R Q 1)$. For one unit increase in the level of privacy awareness (aware), the odds of an accept decision decreases by a factor of $0.45(p<.001)$. This indicates that a higher level of privacy awareness makes people more concerned about potential privacy violations (e.g., via undesired inferences), leading to a rejection of the service.

Utility-privacy tradeoff. Utility-privacy tradeoff was also a significant decision factor since it can act as a proxy for assessing how the IoT service would be beneficial (or risky). In this analysis, we treated this factor as a continuous variable (atomic vectors) in order to ease interpretation: (1) Benefits $\gg$ Risks, (2) Benefits $>$ Risks, (3) Benefits $\approx$ Risks, (4) Benefits $<$ Risks, (5) Benefits $\ll$ Risks. As it can be seen, a higher value of utilityprivacy tradeoff indicates that people consider a specific service to present more risks than benefits. We found that people decided not to use the IoT service if they felt it was risky; for one unit increase in the level of perception of privacy risks relative to utility benefits (tradeoff), the probabilities of accepting the service decrease by a factor of 0.13 $(p<0.001)$.

Privacy segment. Regarding privacy propensity, privacy segment information is shown to be a significant user-specific factor impacting people's privacy decisions. As discussed earlier, we discovered three privacy segments and assigned each participant with one of the resulting segments. However, the regression results showed that not all segments are associated with the DV at the significance level of 0.05 . Nevertheless, we could verify that a segment labeled as VS to privacy can be clearly distinguished from a somewhat insensitive (SI) segment. As expected, users who are clustered into the VS segment tend to make more conservative decisions than others in the SI segment (baseline). For people under VS segment (segment:VerySensitive), the odds of an accept decision are predicted to be 0.78 times lower than people under SI segment $(p<0.001)$.

Privacy self-efficacy. Privacy self-efficacy also acted as an important decision factor as described in $[30,35]$. Contrary to privacy awareness, a higher level of privacy self-efficacy makes people more permissive to the IoT service; for one unit increase in the level of privacy self-efficacy (efficacy), the odds of using IoT services increase by a factor of 2.59 $(p<0.05)$. This is because people who have high privacy self-efficacy believe that they can protect their privacy themselves against the services capable of revealing personal information.

\subsection{Factors Impacting Confidence in Privacy Decisions}

Even though we figured out how diverse underlying factors impact the type of privacy decisions that people made, it is still unclear whether they were confident about their decisions and what factors contribute to their confidence. Therefore, we measured the level of decision confidence via a 5-pt Likert scale in our survey, along with binary privacy decisions.

Since the measured confidence level is ordinal in nature, we used a CLMM [70] for identifying factors related with the user's confidence in his/her privacy decisions. CLMM, a sort of linear mixed 
models (LMM), is designed to handle the ordered but non-continuous ordinal response data, such as ours. Like GLMM, it can take random subject effects into account for multiple measurements taken on the same individual or across time.

4.2.1 Problem Definition. Modeling privacy decision confidence can be generally defined as follows. Consider a CLMM model with $p$ covariates for the cumulative probability of the ordinal response $Y_{i j}$ of the subject $i(1, \ldots, N)$ for the item $j\left(1, \ldots, n_{i}\right)$ falling in the category $k$ or below $(k=1, \ldots, K-1)$ :

$$
\operatorname{Logit}\left(P\left(Y_{i j} \leq k\right)\right)=\theta_{k}-x_{i j}^{T} \beta-v_{i},
$$

where $Y_{i j}$ is the confidence level of the privacy decision made by the $i$ th subject toward the $j$ th scenario ( 1 : very unconfident, $\ldots, 5$ : very confident); $\theta_{k}$ is a set of threshold parameters (cut-points) for the response categories, $x_{i j}$ is the $(p+1)$-dimensional vector of IVs; $\beta$ is the $(p+1)$-dimensinal vector of regression coefficients; $v_{i}$ is the random subject effects distributed iid-normal: $v_{i} \sim \mathcal{N}\left(0, \sigma_{v}^{2}\right)$; $N$ is the number of subjects (488); and $n_{i}$ is the number of scenarios shown to the $i$ th subject (15); and $K$ is the number of response categories (5). We specified a logit link function since we assumed that the probability distribution of $Y_{i j} \leq k$ is binomial.

4.2.2 Model Selection. Like the model selection approach described in Section 4.1.2, we performed a backward stepwise elimination based on LRT to find the most parsimonious CLMM model. As a result, we fitted the reduced CLMM model ${ }^{13}$ as shown below:

$$
\begin{aligned}
\operatorname{Logit}\left(P\left(Y_{i j} \leq k\right)\right)= & \theta_{k}-\beta_{1}\left(\text { location }_{i j}\right)-\beta_{2}\left(\text { infer }_{i j}\right)-\beta_{3}\left(\text { aware }_{i j}\right)-\beta_{4}\left(\text { tradeoff }_{i j}\right) \\
& -\beta_{5}\left(\text { segment }_{i}\right)-\beta_{6}\left(\text { efficacy }_{i}\right)-\beta_{7}\left(\text { residence }_{i}\right)-\beta_{8}\left(\text { income }_{i}\right)-v_{i},
\end{aligned}
$$

where $\left\{\right.$ location $_{i j}$, infer $\left.{ }_{i j}\right\}$ are the service location and core inference of the $j$ th scenario shown to the $i$ th subject; $\left\{\right.$ aware $_{i j}$, tradeoff $\left._{i j}\right\}$ are the measured privacy awareness and utility-privacy tradeoff of the $i$ th subject toward the $j$ th scenario; $\left\{\right.$ segment $_{i}$, efficacy $\left.{ }_{i}\right\}$ are the determined privacy segment and self-efficacy of the $i$ th subject; and $\left\{\right.$ residence $_{i}$, income $\left._{i}\right\}$ are the length of US residence and annual household income of the $i$ th subject (i.e., demographic information). $\beta_{p}$ is a regression coefficient of the $p$ th IV $(p=1, \ldots, 8) . Y_{i j}, \theta_{k}$, and $v_{i}$ are the same as denoted by Equation (3).

4.2.3 Interpretation. We presented the regression results of the fitted CLMM model in Table 8. As we did in Section 4.1.3, we interpreted these results primarily based on factors that have a significant association $(p<0.05)$ with the DV (decision confidence). In doing this, we used the same reference category for each factor as described before.

Contextual information. Considering contextual factors, we verified that the service location and inference of personal information significantly impact people's confidence levels in their privacy decisions. We elaborated detailed interpretations in Appendix A.5 to focus on the results related to the research question that we investigate.

Privacy awareness. We previously showed that privacy awareness is a significant factor determining the type of privacy decisions people make. Here, we confirmed that this factor also has influences on how confident they are on their decisions. Our regression results indeed showed that people with high privacy awareness could better assess the privacy implications of the IoT service before using it, thereby making more confident

\footnotetext{
${ }^{13}$ We used ordinal package (https://cran.r-project.org/web/packages/ordinal/), an R implementation of CLMM, for modeling privacy decision confidence.
} 
Table 8. Regression Results of CLMM Model

\begin{tabular}{|c|c|c|c|c|c|}
\hline Factor Impacting Privacy Decision Confidence & Coef $\beta$ & $\operatorname{SE}(\beta)$ & $\mathrm{Z}$ & $p$ & OR $(95 \% \mathrm{CI})$ \\
\hline location:Private & \multicolumn{5}{|c|}{ baseline } \\
\hline location:Work & -0.36 & 0.13 & -2.86 & $* *$ & $\mathbf{0 . 7 0}(0.54-0.89)$ \\
\hline location:Public & -0.39 & 0.13 & -3.08 & $* *$ & $\mathbf{0 . 6 8}(0.53-0.87)$ \\
\hline infer:DeviceID $\Rightarrow$ UserLocation & \multicolumn{5}{|c|}{ baseline } \\
\hline infer:Electricity $\Rightarrow$ EnergyConsumptionPattern & -0.16 & 0.17 & -0.95 & & $0.85(0.61-1.19)$ \\
\hline infer:Motion $\Rightarrow$ Presence & -0.02 & 0.17 & -0.13 & & $0.98(0.71-1.36)$ \\
\hline infer:Video $\Rightarrow$ Presence & 0.28 & 0.13 & 2.22 & $*$ & $1.33(1.03-1.71)$ \\
\hline infer: Voice $\Rightarrow$ DeviceControlIntention & -0.14 & 0.17 & -0.82 & & $0.87(0.62-1.21)$ \\
\hline infer: Voice $\Rightarrow$ Identity & -0.49 & 0.17 & -2.93 & $* *$ & $\mathbf{0 . 6 1}(0.44-0.85)$ \\
\hline infer:Photo $\Rightarrow$ Identity & 0.20 & 0.10 & 1.95 & & $1.22(1.00-1.50)$ \\
\hline infer:Video $\Rightarrow$ Identity & 0.18 & 0.13 & 1.41 & & $1.20(0.93-1.54)$ \\
\hline infer:Video $\Rightarrow$ PhysicalActivity & 0.40 & 0.13 & 3.19 & $* *$ & $1.50(1.17-1.92)$ \\
\hline infer:Photo $\Rightarrow$ Emotion & 0.23 & 0.17 & 1.35 & & $1.26(0.90-1.76)$ \\
\hline infer: $\mathrm{OBD} \Rightarrow$ Nothing & -0.38 & 0.17 & -2.26 & $*$ & $\mathbf{0 . 6 8}(0.49-0.95)$ \\
\hline infer:Vital $\Rightarrow$ Nothing & -0.33 & 0.17 & -1.93 & & $0.72(0.52-1.01)$ \\
\hline aware & 0.69 & 0.13 & 5.27 & **** & $2.00(1.55-2.59)$ \\
\hline tradeoff & 0.49 & 0.03 & 17.82 & $* * *$ & $1.64(1.55-1.73)$ \\
\hline segment: SomewhatInsensitive & \multicolumn{5}{|c|}{ baseline } \\
\hline segment: SomewhatSensitive & -0.35 & 0.22 & -1.62 & & $0.70(0.46-1.08)$ \\
\hline segment:VerySensitive & 1.21 & 0.18 & 6.77 & $* * *$ & $3.34(2.36-4.73)$ \\
\hline segment:Undecided & 0.41 & 0.24 & 1.72 & & $1.50(0.95-2.39)$ \\
\hline efficacy & 1.41 & 0.38 & 3.76 & $* * *$ & $4.09(1.96-8.54)$ \\
\hline residence:00--04 & \multicolumn{5}{|c|}{ baseline } \\
\hline residence:05--10 & -5.62 & 1.98 & -2.84 & $* *$ & $\mathbf{0 . 0 0}(0.00-0.18)$ \\
\hline residence: $11--20$ & -5.85 & 1.94 & -3.02 & $* *$ & $\mathbf{0 . 0 0}(0.00-0.13)$ \\
\hline residence: $>20$ & -5.17 & 1.83 & -2.83 & $* *$ & $\mathbf{0 . 0 1}(0.00-0.21)$ \\
\hline residence: $\mathrm{n} / \mathrm{a}$ & -3.40 & 2.07 & -1.64 & & $0.03(0.00-1.94)$ \\
\hline income: $<\$ 20,000$ & \multicolumn{5}{|c|}{ baseline } \\
\hline income: $\$ 20,000--\$ 34,999$ & -0.77 & 0.27 & -2.89 & $* *$ & $\mathbf{0 . 4 6}(0.27-0.78)$ \\
\hline income: $\$ 35,000--\$ 49,999$ & -0.61 & 0.27 & -2.27 & $*$ & $\mathbf{0 . 5 4}(0.32-0.92)$ \\
\hline income: $\$ 50,000--\$ 74,999$ & -0.51 & 0.26 & -1.98 & $*$ & $\mathbf{0 . 6 0}(0.36-1.00)$ \\
\hline income: $\$ 75,000--\$ 99,999$ & -0.46 & 0.28 & -1.64 & & $0.63(0.36-1.10)$ \\
\hline income: $>\$ 100,000$ & -0.21 & 0.31 & -0.69 & & $0.81(0.44-1.48)$ \\
\hline income: $n / a$ & -2.54 & 0.62 & -4.11 & $* * *$ & $\mathbf{0 . 0 8}(0.02-0.27)$ \\
\hline
\end{tabular}

Note: $* p<0.05, * * p<0.01, * * * p<0.001$.

decisions (RQ2). For one unit increase in the level of awareness of possible inferences of personal information by IoT services (aware), the cumulative probabilities of making confident privacy decisions increase by a factor of $2(p<0.001)$.

Utility-privacy tradeoff. We obtained a similar result about utility-privacy tradeoff; each one unit increase in the perception of privacy risks relative to utility benefits (tradeoff) will increase the level of decision confidence by a factor of $1.64(p<0.001)$. This means that people tend to make confident decisions if they believe the presented IoT services were risky. In this case, they are probably more cautious about the corresponding service and therefore are forced to make more careful and confident decisions. 
Privacy segment. Regarding privacy propensity, privacy segment has proven to be a significant factor impacting the confidence level of privacy decisions. In our previous GLMM analysis, we have confirmed that privacy-conscious people tend to make conservative decisions. And now, we found out that these people make such decisions with high confidence. For people who are VS to privacy issues in IoT (segment:VerySensitive), the cumulative odds of making confident decisions to accept (or reject) the service are predicted to be $234 \%$ higher than those who are indifferent to privacy $(p<0.001)$.

Privacy self-efficacy. We also realized that privacy self-efficacy can be considered as a rough estimate of people's confidence in their decisions. For one unit increase in the level of privacy self-efficacy (efficacy), the cumulative odds of confident privacy decision-making increase by a factor of $4.09(p<0.001)$.

Demographic information. We uncovered the fact that some demographic information, such as the residence period in the US and annual income level, impacts people's decision confidence as well. Interestingly, a minority of people are more confident about making privacy decisions. To begin with, people who have lived in the US more than at least 5 years (e.g., residence: $>20$ ) are about $100 \%$ less likely to make confident decisions compared to those whose residence period is less than 4 years $(p<0.01)$. It indicates that people who recently moved to the US are generally knowledgeable about the properties of the IoT service, which possibly can invade their privacy. Next, we found that people at the higher income level tend to make less confident decisions than low-income people. There is specifically a $40 \%$ chance of making a confident decision for people whose annual household income falls between $\$ 50,000$ and $\$ 74,999$ (income : $\$ 50,000--\$ 74,999$ ) than people who earn less than $\$ 20,000$ annually $(p<0.05)$. This means that low-income US residents are more aware of the potential privacy risks in the scenarios than others, thereby trying to make decisions as confidently as possible. This finding is consistent with [46] even though this study is targeted at traditional online privacy, not IoT privacy.

Through the abovementioned statistical analysis, we presented what and how underlying factors are associated with people's privacy decisions and their confidence in such decisions. We specifically confirmed that having a higher level of privacy awareness is positively correlated with the probabilities of making more conservative (RQ1) and confident (RQ2) decisions, namely better-informed privacy decision-making in IoT.

\section{PREDICTING PRIVACY DECISIONS IN IOT}

In this section, we address the implications of the informed decision-making in the view of privacy decision support. We first investigate whether it is feasible to build ML models that accurately predict people's decisions based on their observed privacy behaviors. Additionally, we also analyze the relative importance of the input features (privacy decision factors) used to train the ML models. Most importantly, we validate whether privacy decisions made with more confidence can yield the model with better predictive performance. We had observed in a previous situated survey study that people's privacy decisions toward hypothetical IoT scenarios often seem unconfident (i.e., random choice). We suspect that these decisions would not be useful for predicting their future decisions due to the lack of consistent behavioral patterns. We are therefore specifically interested in the following research question:

RQ3: Will privacy decisions made with confidence form a better basis for accurately predicting people's preferred privacy decisions toward unseen IoT services (effective privacy decision support)? 


\subsection{Feature Importance on Privacy Decision Prediction}

We used RF, an ensemble learning method for classification, as an algorithm for predicting privacy decisions. We chose RF because previous research showed that it was effective in helping improve the accuracy of user-defined privacy policies [64] but also known to be effective at generalizing to variants not seen in the training data (less prone to over-fitting), unlike other popular algorithms such as decision trees [7]. Another advantage of RF is that the trained model can compute the relative importance of the input features in making a prediction. The feature importance can provide us with practical insights for constructing ML models for privacy decision support.

5.1.1 Experimental Setup. We trained the RF model ${ }^{14}$ by using all attributes (features) described in Table 6 with the following exceptions. We excluded subject ID because we do not need to differentiate each participant (currently focusing on a one-size-fits-all model). We also excluded decision confidence as an input feature since we rather investigate the performance of the models trained on multiple datasets divided by decision confidence. In addition, we excluded utility-privacy tradeoff since it is strongly correlated with privacy decisions (see Section 4.1.3). Even though utility-privacy tradeoff could act as an important feature (semi-final decision), it is unrealistic to ask users to mark the ordinal values of utility-privacy tradeoffs whenever a prediction is to be generated. As a result, we came up with 16 input features for training the RF model.

Regarding a performance metric, we primarily used the area under the ROC curve (AUC) because it is unaffected by the class imbalance problem (there were about $40 \%$ accept and $60 \%$ reject decisions in our dataset); AUC is independent of the threshold applied to compute the probability of the binary classification results. Additionally, AUC itself is comprehensible; a random classifier has an AUC of 0.5 while a perfect classifier has an AUC of 1.0. Since our dataset is small $(7,320$ instances) and sparse ( $92 \%$ of the user-scenario matrix was empty $\left.{ }^{15}\right)$, we evaluated the trained models through 10 -fold cross validation $(\mathrm{CV})$ for deriving a more accurate estimate of their prediction performance.

5.1.2 Experiment Results. The mean AUC of the trained RF model across all folds was $76.41 \%$ $( \pm 3.03 \%)$. Even though this performance was not too bad (clearly better than random), it should be improved since even a single false prediction may cause undesired inferences of personal information, making users reluctant to follow privacy recommendations. We will discuss how we can achieve better predictive performance in a later section. Figure 2 displays the relative importance of the input features for the trained RF model. We used a randomly chosen $80 \% / 20 \%$ training/test set to compute the feature importance. The results showed that personal privacy propensity (overall privacy awareness, privacy self-efficacy, privacy segment; listed in order of importance) is crucial in predicting people's privacy decisions in IoT. Furthermore, we found that user features (e.g., income, age) were more important than scenario features (e.g., core inference, data retention).

\subsection{Decision Confidence and Predictive Performance}

Since the decision-maker's confidence is a critical component in making rational judgments under uncertainty [21], we hypothesize that privacy decisions made with confidence show more clearly identifiable and predictable behavioral patterns of the user than unconfident decisions, including random choices. Accordingly, we assumed that an ML model trained on confident decisions would better capture these patterns, leading to superior predictive performance $(R Q 3)$.

\footnotetext{
${ }^{14}$ We used scikit-learn (https://scikit-learn.org/), python implementation of RF, for all of our ML experiments.

${ }^{15}$ Each participant responded to about $8 \%$ of all available scenarios.
} 


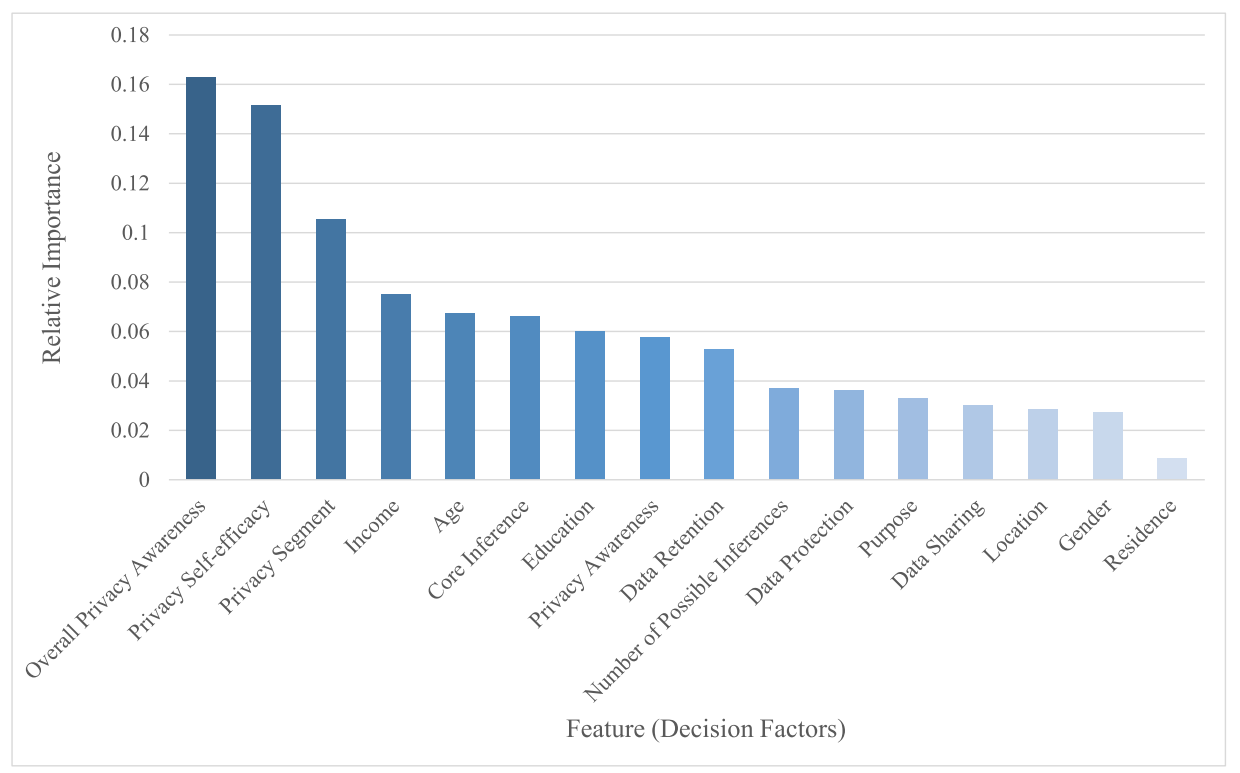

Fig. 2. Feature importance (Random forest; $80 \% / 20 \%$ random train/test split).

Table 9. Data Size and Class Distribution

\begin{tabular}{ccccc}
\hline Confidence Level & \# Instances & \# Accept Decisions & \# Reject Decisions & Accept Ratio \\
\hline Neutral and Unconfident & $\mathbf{1 , 4 0 2}$ & $\mathbf{7 1 5}$ & $\mathbf{6 8 7}$ & $\mathbf{5 1 . 0 0 \%}$ \\
Very Unconfident (1) & 69 & 18 & 51 & $26.09 \%$ \\
Unconfident (2) & 286 & 146 & 140 & $51.05 \%$ \\
Neutral (3) & 1,047 & 551 & 496 & $52.63 \%$ \\
Confident (4) & $\mathbf{3 , 2 5 4}$ & $\mathbf{1 , 5 7 7}$ & $\mathbf{1 , 6 7 7}$ & $\mathbf{4 8 . 4 6 \%}$ \\
Very Confident (5) & $\mathbf{2 , 6 6 4}$ & $\mathbf{6 2 4}$ & $\mathbf{2 , 0 4 0}$ & $\mathbf{2 3 . 4 2 \%}$ \\
Total & 7,320 & 2,916 & 4,404 & $39.84 \%$ \\
\hline
\end{tabular}

5.2.1 Experimental Setup. In order to answer $R Q 3$, we first divided our dataset by the five levels of decision confidence (see Table 9). The number of unconfident (286) and very unconfident (69) decisions is very small compared to other types of decisions, and it is difficult to obtain a meaningful predictive performance with models trained on such a small amount of data. Therefore, we merged them with neutral $(1,047)$ decisions into a neutral and unconfident $(1,402)$ category. We finally trained three separate RF models based on these datasets (boldfaced rows in Table 9). Regarding the performance measurement, we used the mean AUC computed through 10-fold CV. We also presented classification accuracy, which is the number of correct predictions made divided by the total number of predictions made, as reference.

5.2.2 Experiment Results. Figure 3 presents the comparison of predictive performance of all the trained RF models; it clearly demonstrates that the performance increases with the level of decision confidence. Especially the RF model exclusively trained on very confident decisions resulted in the highest mean $\mathrm{AUC}^{16}$ score of $86.87 \%$ (best model), which represents an increase of about

\footnotetext{
${ }^{16} \mathrm{AUC}$ is a performance metric, which is immune to the class distribution of the given training set $(23 \%$ vs. $40 \%$ accept ratio in very confident and all available decisions). AUC is considered as a more rigorous metric than accuracy.
} 


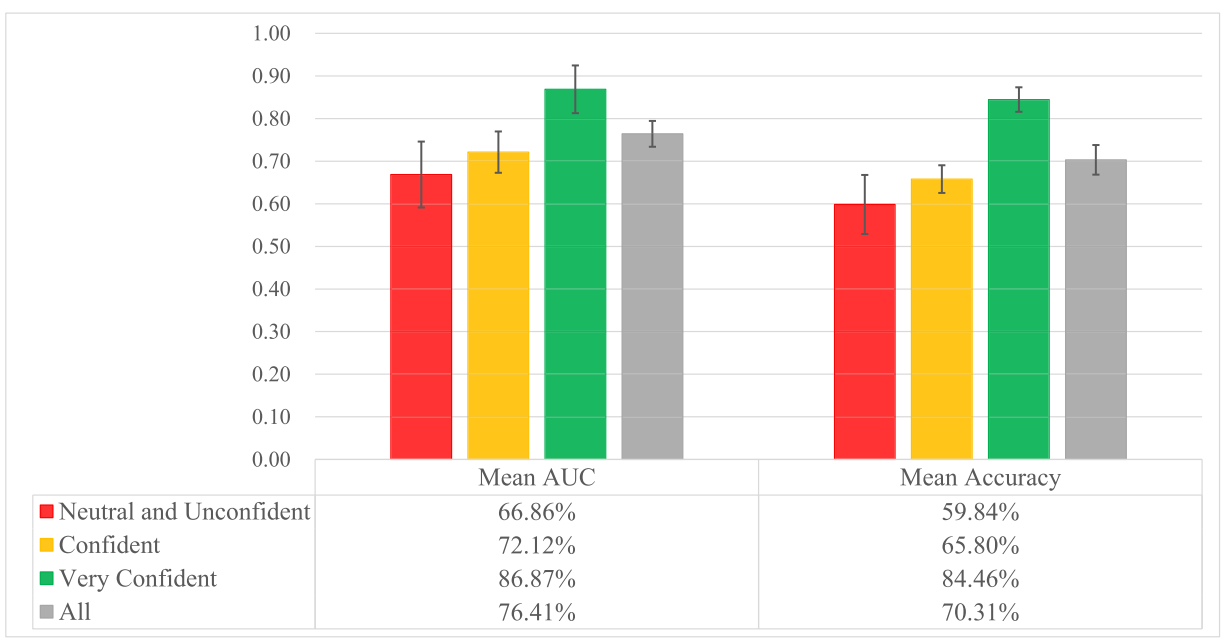

Fig. 3. Predictive performance per confidence level (Random forest; 10 -fold cross validation). Note: Error bars represent two standard deviations that account for about $95 \%$ of the mean AUC/accuracy.

$10 \%$ to the baseline model trained on all available decisions. The difference to all other models is statistically significant (paired $t$-test with Holms-Bonferroni correction; $p<0.05$ ). This is an interesting finding because the size of training data used to construct the best model is approximately $64 \%$ smaller than the baseline model (see Table 9). This result implies that we may be able to build a highly accurate privacy recommendation engine even with a relatively small amount of human behavioral data, if we could ascertain that the data was collected from users who decide with confidence. On the other hand, the model based on neutral and unconfident decisions ranked the lowest $(66.86 \%)$ with the largest variations $( \pm 7.73 \%)$ in the AUC scores calculated for each fold. The measurement of classification accuracy came up with similar results (see the right part of Figure 3). To illustrate the relationships between decision confidence and model performance more clearly, we drew ROC curves for each trained RF model (see Figure 4). An ROC curve is constructed by plotting the trained model's true positive rate $(T P R)$ against the false positive rate $(F P R)$ for all the possible decision thresholds $([0.0,1.0])$. In other words, the ROC curve shows the tradeoff between sensitivity $(T P R)$ and specificity $(1-F P R)$. As a baseline, a random classifier is expected to draw a 45-degree diagonal line $(T P R=F P R)$. The closer the curve comes to the top left corner of the ROC space, the more accurate the classifier is, and vice versa. Note that AUC is the two-dimensional area underneath the entire ROC curve. It is also equivalent to the probability that the classifier will rank a randomly chosen positive example higher than a randomly chosen negative example.

As already mentioned, the classifier trained on highly confident decision samples showed the best performance across all possible classification thresholds (Figure 4(c)), not only due to their AUC but also due to their small variance. By contrast, the prediction of the classifier based on unconfident (or at least neutral) decisions was not only the most inaccurate but the most unreliable (Figure 4(a)). The classifier that learned all available privacy decisions showed a reasonable performance with minimum variance (Figure 4(d)). This is because the largest dataset was used to construct this prediction model.

Considering all the results of our experiments, we argue that it is important to put as many confident decision-making instances as possible into the ML model in order to maximize its predictive performance (RQ3). To that end, it is necessary to build systems that help users in a user-friendly 


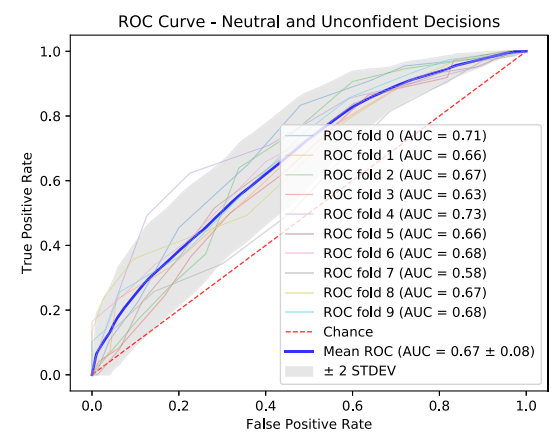

(a) Neutral and Unconfident Decisions

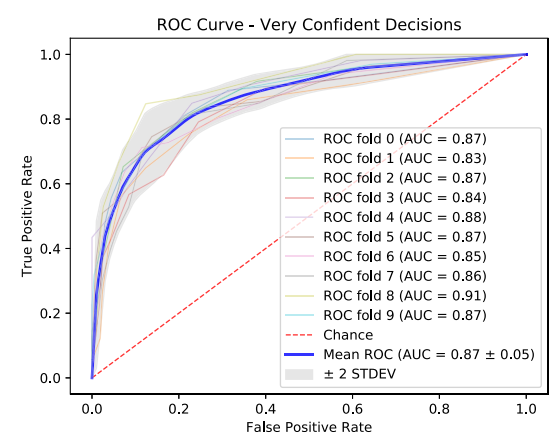

(c) Very Confident Decisions

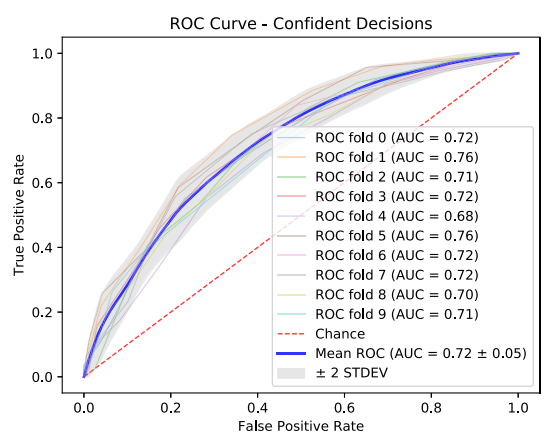

(b) Confident Decisions

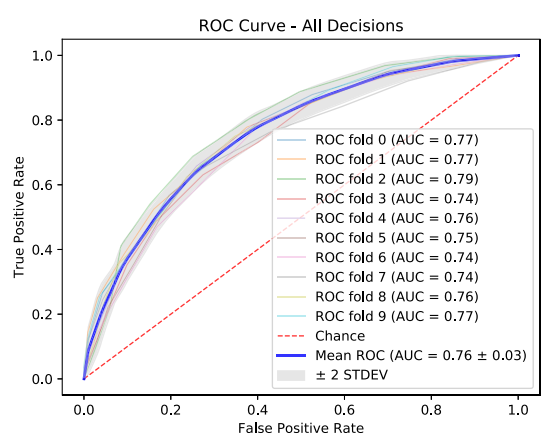

(d) All Decisions

Fig. 4. Receiver operating characteristic (ROC) curve.

way to make more confident decisions. We will describe recommendations for such PAS in the next section.

\section{RESULTS}

In this section, we present the main takeaway we have gained from our data analysis. First, we additionally confirm the causal relationships between privacy awareness and decision confidence, which further supports our claim that making people aware of privacy risks allows them to make more confident decisions $(R Q 2)$. As discussed in Section 5.2, enhancing user confidence in privacy decision-making is one of our research goals since it can enable highly effective privacy decision support. In pursuit of this goal, we then propose functional requirements for PAS to maximize IoT users' privacy awareness, thereby enabling them to make more confident privacy decisions (RQ3). We also present a concrete example of how we can implement PAS in IoT. Furthermore, we describe ways to build a highly optimized privacy decision support system from the perspective of ML.

\subsection{Privacy Awareness and Decision Confidence}

In our statistical analysis, we confirmed that privacy awareness is positively associated with decision confidence. However, this does not necessarily mean they have explicit causal relationships. We performed a path analysis based on structural equation modeling (SEM) ${ }^{17}$ to uncover

\footnotetext{
${ }^{17}$ We used lavaan package (http://lavaan.ugent.be/), an R implementation of SEM, for performing path analysis.
} 


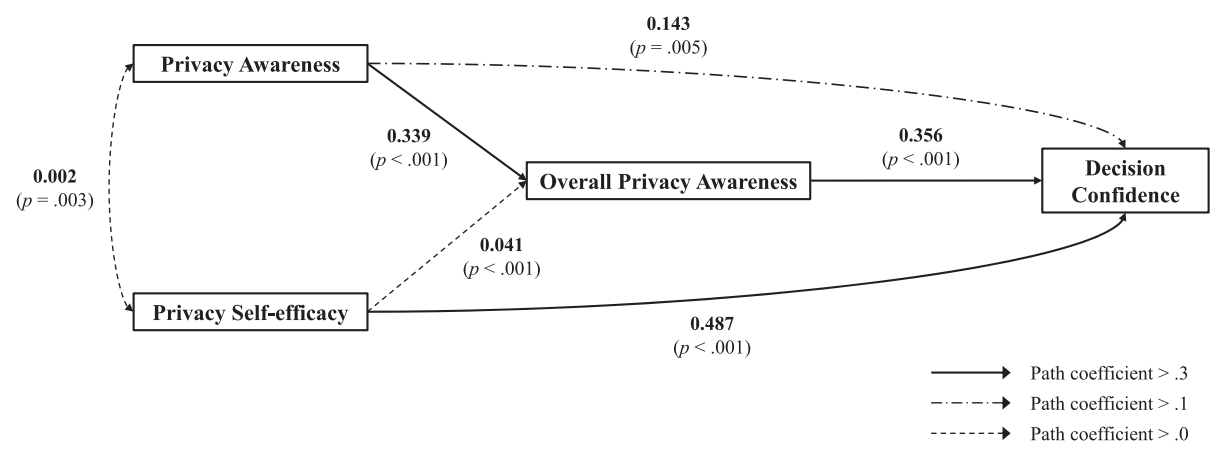

Fig. 5. Path analysis of decision confidence.

any causal relations between (overall) privacy awareness, privacy self-efficacy, and decision confidence. We verified that there exists a strong chain of causation between privacy awareness, overall privacy awareness, and decision confidence (path coefficient $>0.3$, see Figure 5). Note that privacy self-efficacy also has a strong causal relationship with decision confidence, but not with (overall) privacy awareness; this is probably because privacy self-efficacy measures self-perception (which might be delusional), while privacy awareness measures true knowledge. Even though there exists a causal link between privacy self-efficacy and decision confidence, it is not easy to exploit this relationship since user-perceived efficacy does not change in a short period of time. Thus, we claim that increasing the level of privacy awareness of the users is a sufficient condition for nudging them to make more confident privacy decisions.

\subsection{Advanced Privacy-Aware System}

6.2.1 Requirements. To augment users' privacy awareness as much as possible, we propose functional requirements to better design and develop PAS in IoT environments. We suggest that PAS should inform users about both collectible sensor data and inferable personal information, as well as how the latter is inferred from the former. This is because we showed that both the type and confidence of privacy decisions are greatly impacted by inference information.

To that end, (1) developers of PAS first need to collaborate closely with IoT service providers to retrieve relevant information regarding the inference. For instance, a service provider of the Wi-Fi-based occupancy monitoring system can explicitly describe how they collect data and infer personal information (here, user presence) because they have an ownership of the system.

Service providers may not describe all the possible inferences from specific sensor data. For instance, a service provider who is utilizing facial recognition software for the purpose of user authentication might be unaware of the fact that a similar technique can be used to infer users' sexual orientation from previously collected image data. Even if they are aware, the service provider is likely not to reveal this additional inference if they do not rely on it for operating the service. Nevertheless, for the sake of user privacy, the user should know the possibility of this inference [12]. Therefore, (2) developers of PAS may want to construct and update a knowledge base about the relationships between sensor data and inferable personal information. Specifically, the knowledge base should be continuously updated when a new inference technique becomes possible through recent technological advancement. This can be done by either manually (e.g., human experts) or possibly automatically (e.g., information retrieval system) analyzing literature relating to data mining and ML. It is also possible that different developers of PAS as well as the research community collaboratively maintain a single up-to-date knowledge base by sharing their knowledge with each other. 


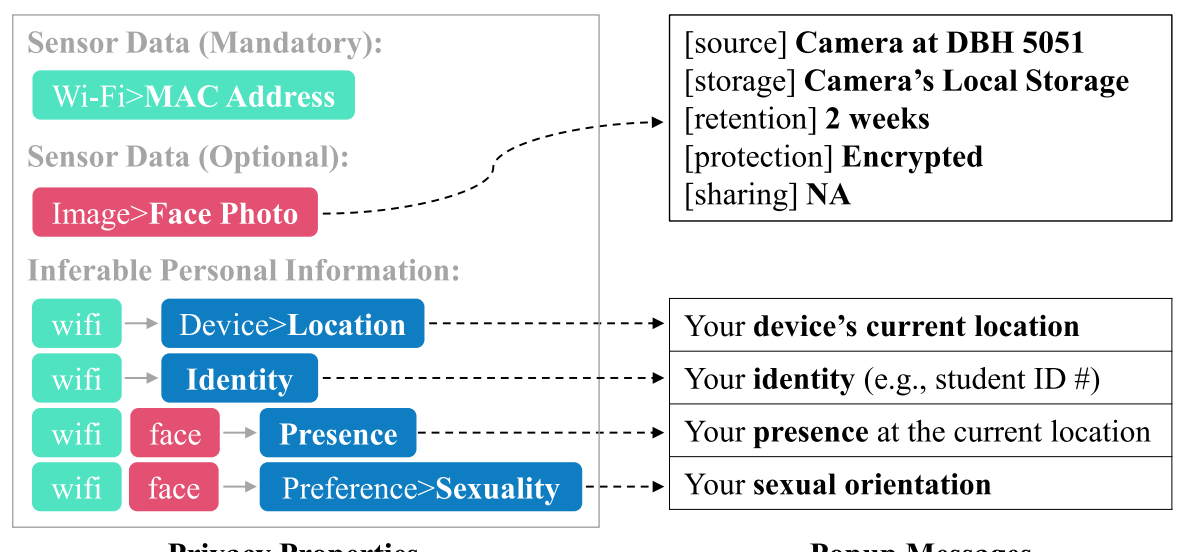

Privacy Properties

Popup Messages

Fig. 6. Example UI of privacy-aware system.

Next, PAS should deliver inference information to users in case they have no effective ways to communicate with an IoT service (i.e., privacy risk communication [11]). Regarding this, (3) PAS should have UIs that convey factual information about potential inferences in a user-friendly manner. For instance, a graphical representation of the detailed procedures of both sensor data collection and personal information inference would be preferable for end users over the display of a long textual description $[17,20]$. In addition, it is necessary to identify inferences that meaningful to the user and selectively display these inferences. This is because too much information might cause information overload and habituation, thereby invalidating the enhancement of privacy awareness [59].

6.2.2 Example. Lee et al. proposed one possible UI for PAS in IoT (see Figure 6) [36]. This is a web page summarizing the possible inferences of personal information can be done by a specific IoT service. As can be seen, sensor data and inferable personal information are distinguished through color coding. In addition, all items are visualized as clickable buttons; users are then able to click the button to view the popup window giving detailed explanations about the item (e.g., data privacy policies). Thus, the users may able to grasp potential privacy risks before making a privacy decision. The authors also described the underlying system architecture and details for implementing this UI [36].

6.2.3 Privacy Decision Support. Even though PAS can present users with information that will help them understand some privacy implications of using various IoT services, users still need to configure their privacy settings themselves. However, some users may have difficulties doing so due to limits in their available time, motivation, and cognitive decision-making abilities [1, 67]. Therefore, attempts are being made to integrate privacy decision support into PAS [14] for giving users privacy recommendations. In this context, our goal is to build a ML model as a core part of privacy decision support that learns and predicts users' privacy decision-making behaviors with a reasonable performance.

To achieve this objective, we would suggest that (4) PAS have some mechanisms to gauge each user's overall privacy awareness and privacy self-efficacy, since these factors were the most important features in our ML models. Our GLMM \& CLMM analysis also confirmed that these variables are strongly associated with people's privacy decision-making. Regarding the measurement of overall privacy awareness, developers of PAS may consider utilizing the experience sampling 
method to repeatedly measure users' privacy awareness regarding the current IoT context, and later aggregate these measurements into a representative metric (e.g., modified mean). Given the fact that privacy self-efficacy does not frequently change, PAS may ask users to complete a onetime questionnaire (e.g., the scale by LaRose and Rifon [35]), and perform CFA to assign a level of privacy self-efficacy to each individual user.

\section{LIMITATIONS AND DISCUSSIONS}

Thus far, we showed that making users aware of the privacy implications of using IoT services will guide them to make more conservative and confident decisions, which will be helpful for protecting their privacy by themselves or with the help of privacy decision support systems. Yet, our work still has some issues that need to be considered and addressed.

\subsection{Representativeness of Dataset}

First, we need to consider the representativeness of the survey participants we recruited through Amazon MTurk. The previous research indicates that Amazon MTurkers are more privacysensitive than the general population [28]. This may result in a sampling bias that makes our results less general. Second, we analyzed participants' stated privacy attitudes and decisions on hypothetical IoT scenarios, and not their actual behavior captured in operational IoT environments. Although we tried to make the scenarios as realistic as possible, we do not definitively know how they would actually behave in real-world situations (i.e., intention-behavior gap). Lastly, we did not collect qualitative feedback, which is often useful for gaining insights into under-represented reasons, opinions, and motivations that people may have of their privacy decision-making. It may reveal other research questions or hypotheses that can inform future research directions. Therefore, it is necessary to develop experimental, but operational, IoT systems/services, recruit participants from the general population (e.g., including people who are not familiar with IoT), and gather their privacy decision-making behaviors as well as qualitative feedback about the IoT services that they interact with. We believe that this will provide us with a more comprehensive privacy decision datasets to understand and predict people's privacy decision-making in IoT.

\subsection{Effective Measurement of Privacy Awareness}

We consulted existing literature to define a set of if-then rules specifying possible and impossible inferences of personal information that can be drawn from sensor data available in IoT, and then used these rules to gauge each survey participant's level of privacy awareness. However, we recognize that in practice, our claimed inferences could be open to misinterpretation. To be specific, one of our expert panel members insisted that it was hard to decide whether a specific inference is possible or not since there was no concrete definition of the quality of the inference (e.g., the desired accuracy of the inference results). Therefore, some participants may not accurately respond to our privacy awareness questions, which may yield to an inaccurate measurement. This suggests that the need for an in-depth study investigating the best way to define and express the inference of personal information, particularly in the context of IoT. In addition, we presented every participant with the same set of possible and impossible inferences (answer options) for each scenario. Displaying different answer options based on the correctness of each participant's responses to the previous scenarios (i.e., computerized adaptive testing) might be a quicker and more effective way to measure his/her privacy awareness. Lastly, our approach will necessarily have scalability issues as long as researchers have to manually generate or review the inference rules according to the literature. Considering the rapid advancements in data mining and ML, it might not be feasible to keep these rules updated. One possibility, albeit not ideal, is to build a probabilistic information retrieval system that automatically extracts relationships between entities (e.g., sensor data and 
personal information) in text data (e.g., academic papers). To build such a system, however, we still need labeled training data (i.e., known relationships between sensor data and personal information), which does not exist to our knowledge. Thus, utilizing systems, such as Snorkel, ${ }^{18}$ designed for programmatically generating labeled training datasets from raw data without much human intervention, is one possible strategy to realize the automated creation and maintenance of the inference rules.

\section{CONCLUSION}

In this article, we investigated how people's privacy awareness impacts the type and confidence of their privacy decisions in IoT. Through an online survey $(N=488)$, we collected people's privacy decisions as well as their levels of privacy awareness toward hypothetical IoT services. Through statistical analysis (random-effects model), we confirmed that people who are well aware of the potential privacy risks of using IoT services tend to make conservative and confident decisions. We also validated that confident privacy decisions form a better basis for building ML models (RF) that accurately learn and predict people's preferred decisions toward unseen IoT services. To the best of our knowledge, this is the first attempt to empirically verify the implications of informed privacy decision-making in preserving user privacy, especially in IoT environments. Based on our findings, we also proposed strategies to better design and develop a PAS, which aims not only to increase IoT users' privacy awareness but also to further assist their decision-making through ML-based privacy recommendations.

\section{A APPENDIX}

\section{A.1 Base Scenarios for Privacy Decision Modeling}

In this appendix, we list the textual descriptions and factor values of all base scenarios.

S01. At your home, your smartphone-connected wearable device collects your vital signs (e.g., blood pressure) to let you easily track, monitor, and share data with your doctors, for your health.

-Location: Private

-Purpose: Health

- Core Inference:

* Vital $\Rightarrow$ Nothing

-Non-core Inference(s):

* Vital $\Rightarrow$ Health Information (e.g., risk of diseases)

-Impossible Inference(s):

*Vital $\Rightarrow$ Sexual Orientation (e.g., homosexuality)

* Vital $\Rightarrow$ Social Relationship

S02. At your home, your smart electricity meter collects your electricity usage to infer your energy consumption patterns, thereby suggesting energy saving methods, for your savings.

-Location: Private

-Purpose: Saving

- Core Inference:

* Electricity Usage $\Rightarrow$ Energy Consumption Pattern

- Non-core Inference(s):

* Electricity Usage $\Rightarrow$ Number of Household Members

\footnotetext{
${ }^{18} \mathrm{https} / /$ hazyresearch.github.io/snorkel/.
} 
* Electricity Usage $\Rightarrow$ Presence

* Electricity Usage $\Rightarrow$ Occupancy Pattern

* Electricity Usage $\Rightarrow$ Sleep Pattern

- Impossible Inference(s):

* Electricity Usage $\Rightarrow$ Emotion

* Electricity Usage $\Rightarrow$ Movie Preference

S03. At your home, your smart TV collects your photo to infer your identity, thereby recommending TV shows based on your watching history, for your convenience.

-Location: Private

- Purpose: Convenience

- Core Inference:

* Photo $\Rightarrow$ Identity

- Non-core Inference(s):

* Photo $\Rightarrow$ Demographic Information (e.g., age, race, gender)

* Photo $\Rightarrow$ Emotion

* Photo $\Rightarrow$ Personality Type

* Photo $\Rightarrow$ Sexual Orientation (e.g., homosexuality)

-Impossible Inference(s):

* Not specified

S04. At your home, your smart TV collects your voice to let you control your TV with voice commands (e.g., raise the volume), for your convenience.

- Location: Private

- Purpose: Convenience

- Core Inference:

* Voice $\Rightarrow$ Device Control Intention

- Non-core Inference(s):

*Voice $\Rightarrow$ Identity

*Voice $\Rightarrow$ Demographic Information (e.g., age, race, gender)

* Voice $\Rightarrow$ Emotion

*Voice $\Rightarrow$ Personality Type

- Impossible Inference(s):

* Voice $\Rightarrow$ Physical Activity

S05. At your home, your smart thermostats detect people's motions to infer their presence in the house, thereby automatically adjusting temperature settings, for your savings.

-Location: Private

- Purpose: Saving

- Core Inference:

* Motion $\Rightarrow$ Presence

- Non-core Inference(s):

* Motion $\Rightarrow$ Moving Pattern

* Motion $\Rightarrow$ Occupancy Pattern

* Motion $\Rightarrow$ Sleep Pattern

- Impossible Inference(s):

* Motion $\Rightarrow$ Identity

* Motion $\Rightarrow$ Demographic Information (e.g., age, race, gender)

S06. At your home, your smart voice assistant collects your voice to infer your identity, thereby verifying that you are a registered driver to remotely control your connected car through voice commands (e.g., OK Google, start up my car), for your safety. 
-Location: Private

- Purpose: Safety

- Core Inference:

* Voice $\Rightarrow$ Identity

-Non-core Inference(s):

* Voice $\Rightarrow$ Demographic Information (e.g., age, race, gender)

* Voice $\Rightarrow$ Emotion

* Voice $\Rightarrow$ Personality Type

- Impossible Inference(s):

* Voice $\Rightarrow$ Dietary Habit

* Voice $\Rightarrow$ Sexual Orientation (e.g., homosexuality)

S07. While you are driving your connected car, your vehicle collects its on-board diagnostics (OBD) data (e.g., RPM, speed, pedal position) to let you easily track, monitor, and share data with vehicle maintenance facilities, for your safety.

-Location: Private

-Purpose: Safety

- Core Inference:

* $\mathrm{OBD} \Rightarrow$ Nothing

-Non-core Inference(s):

* $O B D \Rightarrow$ Driving Habit

-Impossible Inference(s):

* $\mathrm{OBD} \Rightarrow$ Social Relationship

* OBD $\Rightarrow$ Political View

* $\mathrm{OBD} \Rightarrow$ Religion

S08. While you are driving your connected car, your vehicle collects your photo to infer your emotion, thereby giving real-time alerts if you are emotionally unstable while driving, for your safety.

-Location: Private

-Purpose: Safety

- Core Inference:

* Photo $\Rightarrow$ Emotion

-Non-core Inference(s):

* Photo $\Rightarrow$ Identity

* Photo $\Rightarrow$ Demographic Information (e.g., age, race, gender)

* Photo $\Rightarrow$ Personality Type

* Photo $\Rightarrow$ Sexual Orientation (e.g., homosexuality)

-Impossible Inference(s):

* Photo $\Rightarrow$ Financial Condition

S09. At your workplace, your employer's face recognition device collects your photo to infer your identity, thereby allowing you to freely enter the building without a badge, for your convenience.

-Location: Workplace

-Purpose: Convenience

- Core Inference:

* Photo $\Rightarrow$ Identity

-Non-core Inference(s):

* Photo $\Rightarrow$ Presence

* Photo $\Rightarrow$ Attendance Pattern 
* Photo $\Rightarrow$ Emotion

* Photo $\Rightarrow$ Personality Type

* Photo $\Rightarrow$ Sexual Orientation (e.g., homosexuality)

-Impossible Inference(s):

* Photo $\Rightarrow$ Cognitive Activity

* Photo $\Rightarrow$ Energy Consumption Pattern

S10. At your workplace, your employer's network devices collect a unique ID of your mobile device (e.g., Wi-Fi MAC address of your phone) to infer your location, thereby helping you find a specific meeting room, for your convenience.

-Location: Workplace

- Purpose: Convenience

-Core Inference:

* Device ID $\Rightarrow$ User Location

- Non-core Inference(s):

* Device ID $\Rightarrow$ Identity

* Device ID $\Rightarrow$ Moving Pattern

- Impossible Inference(s):

* Device ID $\Rightarrow$ Emotion

* Device ID $\Rightarrow$ Physical Activity

S11. At your workplace, your employer's smart CCTVs collect your video to infer your physical activity (e.g., types of physical movement) inside the building, thereby providing you personalized health recommendations (e.g., you need to walk at least 30 minutes per day), for your health.

-Location: Workplace

-Purpose: Health

- Core Inference:

* Video $\Rightarrow$ Physical Activity

- Non-core Inference(s):

*Video $\Rightarrow$ Presence

*Video $\Rightarrow$ Attendance Pattern

*Video $\Rightarrow$ Social Relationship

*Video $\Rightarrow$ Moving Pattern

* Video $\Rightarrow$ Health Information (e.g., risk of diseases)

- Impossible Inference(s):

* Video $\Rightarrow$ Cognitive Activity

* Video $\Rightarrow$ Political View

S12. At your workplace, your employer's smart CCTV collects your video to infer your presence at your office, thereby automatically managing your attendance (i.e., auto-timesheet), for your convenience.

-Location: Workplace

- Purpose: Convenience

- Core Inference:

* Video $\Rightarrow$ Presence

- Non-core Inference(s):

* Video $\Rightarrow$ Attendance Pattern

*Video $\Rightarrow$ Social Relationship

*Video $\Rightarrow$ Physical Activity

*Video $\Rightarrow$ Smoking Habit 
-Impossible Inference(s):

* Not specified

S13. At a public airport, US government's face recognition device collects your photo to infer your identity, thereby expediting identity verification process with your machine-readable passport, for your convenience.

-Location: Public

- Purpose: Convenience

- Core Inference:

* Photo $\Rightarrow$ Identity

- Non-core Inference(s):

* Photo $\Rightarrow$ Presence

* Photo $\Rightarrow$ Attendance Pattern

* Photo $\Rightarrow$ Emotion

* Photo $\Rightarrow$ Personality Type

* Photo $\Rightarrow$ Sexual Orientation (e.g., homosexuality)

-Impossible Inference(s):

* Not specified

S14. At a public airport, US government's network devices collect a unique ID of your mobile device (e.g., Wi-Fi MAC address of your phone) to infer your location, thereby helping you find a boarding gate, for your convenience.

- Location: Public

-Purpose: Convenience

- Core Inference:

* Device ID $\Rightarrow$ User Location

- Non-core Inference(s):

* Device ID $\Rightarrow$ Identity

* Device ID $\Rightarrow$ Moving Pattern

-Impossible Inference(s):

* Device ID $\Rightarrow$ Personality Type

* Device ID $\Rightarrow$ Health Information (e.g., risk of diseases)

S15. At a public airport, US government's smart CCTVs collect your and others' video to infer their identity, thereby identifying wanted criminals or suspicious people around you, for your safety.

-Location: Public

- Purpose: Safety

- Core Inference:

* Video $\Rightarrow$ Identity

- Non-core Inference(s):

*Video $\Rightarrow$ Presence

*Video $\Rightarrow$ Attendance Pattern

*Video $\Rightarrow$ Social Relationship

*Video $\Rightarrow$ Physical Activity

*Video $\Rightarrow$ Moving Pattern

-Impossible Inference(s):

* Video $\Rightarrow$ Financial Condition

\section{A.2 Recruitment Material and Consent Form}

In this appendix, we first list a recruitment script shown to our survey participants as below. 
- We are conducting an academic survey about the Internet of Things (IoT). We need to understand your opinion about potential privacy issues in using intelligent IoT services. Select the link below to complete the survey. It will take about 15-20 minutes. At the end of the survey, you will receive a code to paste into the box below to receive credit for taking our survey.

- Make sure to leave this window OPEN as you complete the survey-When you are finished, you will return to this page to paste the code into the box.

- The survey link, survey code input form, and submit button were given.

We also present the consent form as below.

- Lead Researcher: Hosub Lee, Donald Bren School of Information and Computer Sciences, UCI (hosubl@uci.edu)

- Faculty Sponsor: Alfred Kobsa, Donald Bren School of Information and Computer Sciences, UCI (kobsa@uci.edu)

- You are asked to participate in a study about Internet of Things (IoT).

- You are eligible to participate if you are at least 18 years or older.

- There are no risks/discomforts with the study.

- There are no direct benefits from participation in the study. However, this study may explain how people react to diverse sensor data collection and personal information inferences being performed by IoT services.

-If you have any comments, concerns, or questions regarding the conduct of this research, please contact the researchers listed above.

- Please contact UCI's Office of Research by phone at (949) 824-7295 or by mail at 141 Innovation Drive, Suite 250, Irvine, CA 92697 if you are unable to reach the researchers and have general questions; have concerns or complaints about the research; have questions about your rights as a research subject; or have general comments or suggestions.

- Participation in this study is voluntary. There is no cost to you for participating. You may refuse to participate or discontinue your involvement at any time without penalty. You are free to withdraw from this study at any time. If you decide to withdraw from this study, you should notify the research team immediately.

- I understand the information provided regarding this study and agree to participate.

- A check-box was given.

\section{A.3 Scenarios for Privacy Segmentation}

In this appendix, we list the three IoT scenarios used for privacy segmentation.

S1. A device of Starbucks (who) takes a photo of you to determine your mood (what). This happens once (persistence), while you are at Starbucks (where), for commercial (reason) purposes, namely to suggest the most suitable product to you based on your mood.

S2. A device of your employer ( who) collects your phone ID (e.g., Wi-Fi MAC address) to verify your identity (what). This happens continuously (persistence), while you are at your workplace (where), for your convenience (reason), namely to automate mundane tasks (e.g., making a payment at the cafeteria).

S3. A device of the government (who) takes a video (what) of you. This happens continuously (persistence), while you are in a public park (where), for your safety (reason), namely to identify wanted criminals around you.

We also present a questionnaire for collecting user reactions to the abovementioned scenarios. 
Q1. Would you want to be notified (notification) about this monitoring?
(1) Yes, always
(2) Yes, but ask me again the next time
(3) No, but ask me again the next time
(4) No, never

Q2. Would you want to allow (permission) this monitoring?
(1) Yes, always
(2) Yes, but ask me again the next time
(3) No, but ask me again the next time
(4) No, never

Q3. How comfortable (comfort) is this monitoring?
(1) Very uncomfortable
(2) Uncomfortable
(3) Somewhat uncomfortable
(4) Neutral
(5) Somewhat comfortable
(6) Comfortable
(7) Very comfortable

Q4. How risky (risk) is this monitoring?
(1) Very risky
(2) Risky
(3) Somewhat risky
(4) Neutral
(5) Somewhat safe
(6) Safe
(7) Very safe

Q5. How appropriate (appropriateness) is this monitoring?
(1) Very inappropriate
(2) Inappropriate
(3) Somewhat inappropriate
(4) Neutral
(5) Somewhat appropriate
(6) Appropriate
(7) Very appropriate

\section{A.4 Contextual Factors Impacting Privacy Decisions}

In this appendix, we explain how each contextual factor impacts people's privacy decisions based on our regression results (see Table 7).

A.4.1 Location. Previous research $[4,37]$ indicates that, in general, people consider IoT-based sensor data collection and analysis as very unacceptable if they occur in private places, like their home. Thus, we interpreted our regression results by considering location: Private as a baseline category. We confirmed that our findings are consistent with the literature. For the IoT service being operated in the workplace (location: Work) and public spaces (location:Public), the odds of using the service (i.e., accept decision) are predicted to be four times and three times higher than the service at the private space, respectively ( $p<0.001$ for both). It also indicates that the US respondents are more used to giving up privacy at their workplaces than public area. 
A.4.2 Core Inference. The type of inference of personal information was also a dominant factor influencing privacy decisions in IoT, as addressed by [38, 51]. Regarding this, we specified a baseline category as users' indoor location inferred from unique identifiers of their mobile devices (infer:DeviceID $\Rightarrow$ UserLocation) as this is the most common inference possible in currently available IoT environments (e.g., Wi-Fi-based occupancy monitoring).

We found that people tend to accept an IoT service if it anonymously infers simple behavioral patterns of theirs. The probabilities of accepting the inference of unidentified individuals' electricity usage patterns (infer:Electricity $\Rightarrow$ EnergyConsumptionPattern) and presence (infer:Motion $\Rightarrow$ Presence) at a certain location are 17 times and 13 times higher than the inference of user location mapped with device IDs, respectively ( $p<0.001$ for both). Similarly, the odds of allowing the voice-based inference of intentions to control consumer electronics ${ }^{19}$ without user identification (infer:Voice $\Rightarrow$ DeviceControlIntention) are more than eight times higher than the baseline inference $(p<0.001)$.

In contrast, people are relatively less receptive toward services involving user identification. The odds of utilizing the service that infers the user's identity are 5.5, 1.6, and 1.3 times higher than the baseline, depending on the type of raw sensor data used: voice (infer:Voice $\Rightarrow$ Identity), photo (infer:Photo $\Rightarrow$ Identity), and video (infer:Video $\Rightarrow$ Identity), respectively $(p<0.001$ for all). We also found that people are more reluctant to admit a video-based identification mechanism than other approaches (photo and voice). Note that inferring users' identity is still considered more acceptable than inferring their current location. This is probably because people are more comfortable with user identification since they already have been using it in non-IoT applications/services (e.g., face unlock on smartphone). However, the inference of their physical activities (e.g., running) based on the captured video data (infer:Video $\Rightarrow$ PhysicalActivity) was unacceptable; the odds to accept this kind of inference are predicted to be 0.51 times lower than the baseline $(p<0.01)$.

Lastly, we intentionally defined IoT services that collect certain sensor data for a specific purpose but does not infer any additional information from it, to check people's reactions to virtually privacy-safe IoT scenarios. As expected, people are more likely to allow the service if it declares not to infer personal information, even though its collectible sensor data might allow sensitive inferences (e.g., health conditions from heart rate measurement). The odds of accepting a service that simply collects $\mathrm{OBD}$ data (infer $: \mathrm{OBD} \Rightarrow$ Nothing) or vital signs (infer: Vital $\Rightarrow$ Nothing) without any inferences are about 15 and 10 times higher than a service that infers user location $(p<0.001$ for both).

A.4.3 Data Sharing. With regard to data privacy policy, people make different privacy decisions depending on whether the collected sensor data will be shared or not. Specifically, people are more likely to refuse to use an IoT service if it shares data with third parties. The odds of using services with data sharing practice (share: Shared) are predicted to be 0.18 times lower compared to other services that do not share data $(p<0.05)$. The fact that virtually all kinds of sensor data might be used to infer higher-level user information caused people to make more conservative decisions. This finding is also aligned with [51].

\section{A.5 Contextual Factors Impacting Confidence in Privacy Decisions}

In this appendix, we explain how each contextual factor impacts people's privacy decision confidence based on our regression results (see Table 8).

A.5.1 Location. Our GLMM analysis has revealed that people tend to reject the IoT services operating in their private spaces. Through this analysis, we further confirmed that they make

\footnotetext{
${ }^{19}$ E.g., Turn on the TV.
} 
such decisions with confidence. To explain, people are $30 \%$ less likely to have high confidence in their privacy decisions toward IoT services at the workplace (location: Work) rather than private spaces like home (location: Private), no matter what decisions made $(p<0.01)$. People showed a similar reaction to the public spaces (location:Public); they make $32 \%$ less confident decisions in public rather than their own places $(p<0.01)$. This is probably because they have limited information (e.g., available sensors) about the services deployed at locations out of their control, therefore they are not fully convinced that they could make a good decision.

A.5.2 Core Inference. Regarding the inference of personal information, we confirmed that people's privacy decision confidence varies depending on the specific types of collectible sensor data, namely video and voice. In general, people make confident decisions about the inferences based on video data. Specifically, the cumulative odds of the level of confidence in privacy decisions being rated in a certain level or above for IoT services inferring users' presence at a specific location through video data captured by surveillance cameras (infer:Video $\Rightarrow$ Presence) are predicted to be $33 \%$ higher than inferring user location based on their unique device IDs (infer:DeviceID $\Rightarrow$ UserLocation), such as Wi-Fi MAC addresses captured by Wi-Fi access points $(p<0.05)$. This indicates that people would be more certain about their decisions if the presented services are collecting sensor data, which can clearly represent their presence in some locations (i.e., video). On the other hand, it is possible that some people do not fully understand how Wi-Fi MAC addresses can be used to infer their current location. In addition, sensor devices collecting video (i.e., cameras) are more easily recognizable in real life, thereby allowing survey participants to better situate themselves in the scenario. In a similar vein, people tend to make confident decisions about the video-based inference of their physical activities (infer: Video $\Rightarrow$ PhysicalActivity); the cumulative odds of having high decision confidence toward this type of inference are $50 \%$ higher than the baseline inference $(p<0.01)$.

In contrast, people are relatively unconfident about the inference based on voice data. The cumulative odds of making a confident privacy decision of whether to accept (or reject) the inference of user identity via speech analysis (infer:Voice $\Rightarrow$ Identity) are predicted to be $39 \%$ lower than location inference based on device IDs $(p<0.01)$. People may perceive voice data itself as a resource that can reveal additional sensitive personal information (e.g., intention, emotion) rather than just identity. Accordingly, it is more difficult for them to make a correct decision if the inference is based on voice data, compared with seemingly primitive data like device IDs.

\section{ACKNOWLEDGMENTS}

The authors would like to thank Yao Li for her valuable comments on the confirmatory factor analysis we performed in this article, and Jihyun Park, Minhaeng Lee, and Jordan Hyungik Oh for their help in defining inference rules.

\section{REFERENCES}

[1] Alessandro Acquisti, Laura Brandimarte, and George Loewenstein. 2015. Privacy and human behavior in the age of information. Science 347, 6221 (2015), 509-514.

[2] Alessandro Acquisti and Jens Grossklags. 2004. Privacy attitudes and privacy behavior. In Economics of Information Security. Springer, 165-178.

[3] Irwin Altman. 1975. The Environment and Social Behavior: Privacy, Personal Space, Territory, and Crowding. Brooks/Cole Pub. Co.

[4] Paritosh Bahirat, Yangyang He, Abhilash Menon, and Bart Knijnenburg. 2018. A data-driven approach to developing IoT privacy-setting interfaces. In Proceedings of the 23rd International Conference on Intelligent User Interfaces (IUI'18). ACM, New York, NY, 165-176. 
[5] Marian Stewart Bartlett, Gwen Littlewort, Mark Frank, Claudia Lainscsek, Ian Fasel, and Javier Movellan. 2005. Recognizing facial expression: Machine learning and application to spontaneous behavior. In Proceedings of the 2005 IEEE Computer Society Conference on Computer Vision and Pattern Recognition (CVPR'05), Vol. 2. IEEE, 568-573.

[6] Douglas Bates, Martin Mächler, Ben Bolker, and Steve Walker. 2014. Fitting linear mixed-effects models using lme4. arXiv:1406.5823.

[7] Yoshua Bengio, Olivier Delalleau, and Clarence Simard. 2010. Decision trees do not generalize to new variations. Computational Intelligence 26, 4 (2010), 449-467.

[8] Mike Bergmann. 2008. Testing privacy awareness. In IFIP Summer School on the Future of Identity in the Information Society. Springer, 237-253.

[9] Igor Bilogrevic, Kévin Huguenin, Berker Agir, Murtuza Jadliwala, Maria Gazaki, and Jean-Pierre Hubaux. 2016. A machine-learning based approach to privacy-aware information-sharing in mobile social networks. Pervasive and Mobile Computing 25 (2016), 125-142. https:/www.sciencedirect.com/science/article/abs/pii/S1574119215000231?via\% 3Dihub.

[10] Timothy A. Brown. 2014. Confirmatory Factor Analysis for Applied Research. Guilford Publications.

[11] L. Jean Camp. 2009. Mental models of privacy and security. IEEE Technology and Society Magazine 28, 3 (2009), 37-46.

[12] Richard Chow. 2017. The last mile for IoT privacy. IEEE Security \& Privacy 15, 6 (2017), 73-76.

[13] Federal Trade Commission et al. 2015. Internet of Things: Privacy \& security in a connected world. Federal Trade Commission, Washington, DC. https://www.ftc.gov/system/files/documents/reports/federal-tradecommission-staff-report-november-2013-workshop-entitled-internet-things-privacy/150127iotrpt.pdf.

[14] Anupam Das, Martin Degeling, Daniel Smullen, and Norman Sadeh. 2018. Personalized privacy assistants for the Internet of Things: Providing users with notice and choice. IEEE Pervasive Computing 17, 3 (2018), 35-46.

[15] Anupam Das, Martin Degeling, Xiaoyou Wang, Junjue Wang, Norman Sadeh, and Mahadev Satyanarayanan. 2017. Assisting users in a world full of cameras: A privacy-aware infrastructure for computer vision applications. In Proceedings of the IEEE Conference on Computer Vision and Pattern Recognition Workshops (CVPRW'17). IEEE, 1387-1396.

[16] André Deuker. 2009. Addressing the privacy paradox by expanded privacy awareness - The example of contextaware services. In IFIP PrimeLife International Summer School on Privacy and Identity Management for Life. Springer, $275-283$.

[17] Serge Egelman, Raghudeep Kannavara, and Richard Chow. 2015. Is this thing on?: Crowdsourcing privacy indicators for ubiquitous sensing platforms. In Proceedings of the SIGCHI Conference on Human Factors in Computing Systems (CHI'15). ACM, 1669-1678.

[18] Gunnar Evermann. 2014. System and method for inferring user intent from speech inputs. US Patent App. 14/298,725.

[19] Ebenezer Hailemariam, Rhys Goldstein, Ramtin Attar, and Azam Khan. 2011. Real-time occupancy detection using decision trees with multiple sensor types. In Proceedings of the 2011 Symposium on Simulation for Architecture and Urban Design. Society for Computer Simulation International, 141-148.

[20] Marian Harbach, Markus Hettig, Susanne Weber, and Matthew Smith. 2014. Using personal examples to improve risk communication for security and privacy decisions. In Proceedings of the SIGCHI Conference on Human Factors in Computing Systems (CHI'14). ACM, 2647-2656.

[21] Brian Hill. 2013. Confidence and decision. Games and Economic Behavior 82 (2013), 675-692. https:// www.sciencedirect.com/science/article/pii/S0899825613001395.

[22] Jason Hong. 2017. The privacy landscape of pervasive computing. IEEE Pervasive Computing 16, 3 (2017), 40-48.

[23] Zhexue Huang. 1997. A fast clustering algorithm to cluster very large categorical data sets in data mining. Data Mining and Knowledge Discovery 3, 8 (1997), 34-39.

[24] Zhexue Huang. 1998. Extensions to the k-means algorithm for clustering large data sets with categorical values. Data Mining and Knowledge Discovery 2, 3 (1998), 283-304.

[25] Carlos Jensen, Colin Potts, and Christian Jensen. 2005. Privacy practices of Internet users: Self-reports versus observed behavior. International fournal of Human-Computer Studies 63, 1-2 (2005), 203-227.

[26] Joseph B. Kadane and Nicole A Lazar. 2004. Methods and criteria for model selection. fournal of the American statistical Association 99, 465 (2004), 279-290.

[27] Dimitri Kanevsky, Joseph Simon Reisinger, Robert Sicconi, and Mahesh Viswanathan. 2012. Hierarchical methods and apparatus for extracting user intent from spoken utterances. US Patent 8,265,939.

[28] Ruogu Kang, Stephanie Brown, Laura Dabbish, and Sara Kiesler. 2014. Privacy attitudes of mechanical turk workers and the U.S. public. In Proceedings of the 10th Symposium On Usable Privacy and Security (SOUPS'14). USENIX Association, 37-49.

[29] Bart Knijnenburg, Alfred Kobsa, and Hongxia Jin. 2013. Dimensionality of information disclosure behavior. International Journal of Human-Computer Studies 71, 12 (2013), 1144-1162.

[30] Alfred Kobsa, Hichang Cho, and Bart Knijnenburg. 2016. The effect of personalization provider characteristics on privacy attitudes and behaviors: An elaboration likelihood model approach. Fournal of the Association for Information Science and Technology 67, 11 (2016), 2587-2606. 
[31] Bastian Könings, Florian Schaub, and Michael Weber. 2013. Who, how, and why? Enhancing privacy awareness in ubiquitous computing. In Proceedings of the 2013 IEEE International Conference on Pervasive Computing and Communications Workshops (PerCom Workshops). IEEE, 364-367.

[32] Irene Kotsia and Ioannis Pitas. 2006. Facial expression recognition in image sequences using geometric deformation features and support vector machines. IEEE Transactions on Image Processing 16, 1 (2006), 172-187.

[33] Marc Langheinrich. 2002. A privacy awareness system for ubiquitous computing environments. In Proceedings of the 4th International Conference on Ubiquitous Computing (UbiComp’02). Springer, 237-245.

[34] Nancy Lankton, D. McKnight, and John Tripp. 2016. Privacy management strategies: An exploratory cluster analysis. In Proceedings of the 22nd Americas Conference on Information Systems (AMCIS'16). Association for Information Systems, 1-10. https://pdfs.semanticscholar.org/3700/49da669cec608be3048376281f93332488fb.pdf.

[35] Robert LaRose and Nora J. Rifon. 2007. Promoting i-safety: Effects of privacy warnings and privacy seals on risk assessment and online privacy behavior. Journal of Consumer Affairs 41, 1 (2007), 127-149.

[36] Hosub Lee, Richard Chow, Mohammad R. Haghighat, Heather M. Patterson, and Alfred Kobsa. 2018. IoT service store: A web-based system for privacy-aware IoT service discovery and interaction. In Proceedings of the 2018 IEEE International Conference on Pervasive Computing and Communications Workshops (PerCom Workshops). IEEE, 107-112.

[37] Hosub Lee and Alfred Kobsa. 2016. Understanding user privacy in Internet of Things environments. In Proceedings of the 2016 IEEE 3rd World Forum on Internet of Things (WF-IoT). IEEE, 407-412.

[38] Hosub Lee and Alfred Kobsa. 2017. Privacy preference modeling and prediction in a simulated campuswide IoT environment. In Proceedings of the IEEE International Conference on Pervasive Computing and Communications (PerCom'17). IEEE, 276-285.

[39] Kuang-Chih Lee, Jeffrey Ho, Ming-Hsuan Yang, and David Kriegman. 2003. Video-based face recognition using probabilistic appearance manifolds. In Proceedings of the 2003 IEEE Computer Society Conference on Computer Vision and Pattern Recognition (CVPR'03), Vol. 1. IEEE, I-I.

[40] Seungwoo Lee, Daye Ahn, Sukjun Lee, Rhan Ha, and Hojung Cha. 2014. Personalized energy auditor: Estimating personal electricity usage. In Proceedings of the IEEE International Conference on Pervasive Computing and Communications (PerCom'14). IEEE, 44-49.

[41] Jialiu Lin, Bin Liu, Norman Sadeh, and Jason Hong. 2014. Modeling users' mobile app privacy preferences: Restoring usability in a sea of permission settings. In Proceedings of the 10th Symposium on Usable Privacy and Security (SOUPS'14). 199-212.

[42] Bin Liu, Mads Schaarup Andersen, Florian Schaub, Hazim Almuhimedi, Shikun (Aerin) Zhang, Norman Sadeh, Alessandro Acquisti, and Yuvraj Agarwal. 2016. Follow my recommendations: A personalized privacy assistant for mobile app permissions. In Proceedings of the 12th Symposium on Usable Privacy and Security (SOUPS'16). USENIX Association, 27-41.

[43] Xiaoming Liu and Tsuhan Cheng. 2003. Video-based face recognition using adaptive hidden Markov models. In Proceedings of the 2003 IEEE Computer Society Conference on Computer Vision and Pattern Recognition (CVPR'03), Vol. 1. IEEE, I-I.

[44] Xiaoxuan Lu, Hongkai Wen, Han Zou, Hao Jiang, Lihua Xie, and Niki Trigoni. 2016. Robust occupancy inference with commodity WiFi. In Proceedings of the 2016 IEEE 12th International Conference on Wireless and Mobile Computing, Networking and Communications (WiMob). IEEE, 1-8.

[45] Chengwen Luo, Long Cheng, Mun Choon Chan, Yu Gu, Jianqiang Li, and Zhong Ming. 2016. Pallas: Self-bootstrapping fine-grained passive indoor localization using WiFi monitors. IEEE Transactions on Mobile Computing 16, 2 (2016), $466-481$.

[46] Mary Madden. 2017. Privacy, Security, and Digital Inequality. Data \& Society, New York.

[47] Kirsten Martin. 2012. Diminished or just different? A factorial vignette study of privacy as a social contract. Fournal of Business Ethics 111, 4 (2012), 519-539.

[48] Kirsten Martin and Helen Nissenbaum. 2016. Measuring privacy: An empirical test using context to expose confounding variables. Columbia Science and Technology Law Review 18 (2016), 176.

[49] Carlo Maria Medaglia and Alexandru Serbanati. 2010. An overview of privacy and security issues in the Internet of Things. In The Internet of Things. Springer, 389-395.

[50] Sharad Mehrotra, Alfred Kobsa, Nalini Venkatasubramanian, and Siva Raj Rajagopalan. 2016. TIPPERS: A privacy cognizant IoT environment. In Proceedings of the IEEE International Conference on Pervasive Computing and Communication Workshops (PerCom Workshops'16). IEEE, 1-6.

[51] Pardis Emami Naeini, Sruti Bhagavatula, Hana Habib, Martin Degeling, Lujo Bauer, Lorrie Cranor, and Norman Sadeh. 2017. Privacy expectations and preferences in an IoT world. In Proceedings of the 13th Symposium on Usable Privacy and Security (SOUPS'17). USENIX Association, 399-412.

[52] Wei Niu, Jiao Long, Dan Han, and Yuan-Fang Wang. 2004. Human activity detection and recognition for video surveillance. In Proceedings of the 2004 IEEE International Conference on Multimedia and Expo (ICME'04), Vol. 1. IEEE, 719-722. 
[53] Patricia A. Norberg, Daniel R. Horne, and David A. Horne. 2007. The privacy paradox: Personal information disclosure intentions versus behaviors. fournal of Consumer Affairs 41, 1 (2007), 100-126.

[54] IEEE Internet of Things et al. 2016. IoT Scenarios. Retrieved April 28, 2016 from https://iot.ieee.org/iot-scenarios.html.

[55] Leysia Palen and Paul Dourish. 2003. Unpacking "privacy" for a networked world. In Proceedings of the SIGCHI Conference on Human Factors in Computing Systems (CHI'03). ACM, New York, NY, 129-136.

[56] Primal Pappachan, Martin Degeling, Roberto Yus, Anupam Das, Sruti Bhagavatula, William Melicher, Pardis Emami Naeini, Shikun Zhang, Lujo Bauer, Alfred Kobsa, Sharad Mehrotra, Norman Sadeh, and Nalini Venkatasubramanian. 2017. Towards privacy-aware smart buildings: Capturing, communicating, and enforcing privacy policies and preferences. In Proceedings of the IEEE 37th International Conference on Distributed Computing Systems Workshops (ICDCSW'17). IEEE, 193-198.

[57] Charith Perera, Rajiv Ranjan, Lizhe Wang, Samee U. Khan, and Albert Y. Zomaya. 2015. Big data privacy in the Internet of Things era. IT Professional 17, 3 (2015), 32-39.

[58] Stefanie Pötzsch. 2008. Privacy awareness: A means to solve the privacy paradox? In IFIP Summer School on the Future of Identity in the Information Society. Springer, 226-236.

[59] Ashwini Rao, Florian Schaub, Norman Sadeh, Alessandro Acquisti, and Ruogu Kang. 2016. Expecting the unexpected: Understanding mismatched privacy expectations online. In Proceedings of the 12th Symposium on Usable Privacy and Security (SOUPS'16). 77-96.

[60] Douglas A. Reynolds and Richard C. Rose. 1995. Robust text-independent speaker identification using Gaussian mixture speaker models. IEEE Transactions on Speech and Audio Processing 3, 1 (1995), 72-83.

[61] Neil Robertson and Ian Reid. 2006. A general method for human activity recognition in video. Computer Vision and Image Understanding 104, 2-3 (2006), 232-248.

[62] Peter H. Rossi and Richard A. Berk. 1985. Varieties of normative consensus. American Sociological Review 50, 3 (1985), 333-347.

[63] Peter H. Rossi and Steven L. Nock. 1982. Measuring Social fudgments: The Factorial Survey Approach. SAGE Publications, Incorporated.

[64] Norman Sadeh, Jason Hong, Lorrie Cranor, Ian Fette, Patrick Kelley, Madhu Prabaker, and Jinghai Rao. 2009. Understanding and capturing people's privacy policies in a mobile social networking application. Personal and Ubiquitous Computing 13, 6 (2009), 401-412.

[65] Sabrina Sicari, Alessandra Rizzardi, Luigi Alfredo Grieco, and Alberto Coen-Porisini. 2015. Security, privacy and trust in Internet of Things: The road ahead. Computer Networks 76 (2015), 146-164. https://www.sciencedirect.com/science/ article/abs/pii/S1389128614003971.

[66] Julius Sim and Chris C. Wright. 2005. The kappa statistic in reliability studies: Use, interpretation, and sample size requirements. Physical Therapy 85, 3 (2005), 257-268.

[67] Daniel J. Solove. 2012. Introduction: Privacy self-management and the consent dilemma. Harvard Law Review 126 (2012), 1880-1903.

[68] Johannes Bernhard Steffens, Egor Valerievich Elagin, Luciano Pasquale Agostino Nocera, Thomas Maurer, and Hartmut Neven. 2001. Face recognition from video images. US Patent 6,301,370.

[69] Xiaoyang Tan, Songcan Chen, Zhi-Hua Zhou, and Fuyan Zhang. 2006. Face recognition from a single image per person: A survey. Pattern Recognition 39, 9 (2006), 1725-1745.

[70] Gerhard Tutz and Wolfgang Hennevogl. 1996. Random effects in ordinal regression models. Computational Statistics \& Data Analysis 22, 5 (1996), 537-557.

[71] Vincent Wan and William M. Campbell. 2000. Support vector machines for speaker verification and identification. In Proceedings of the 2000 IEEE Signal Processing Society Workshop on Neural Networks for Signal Processing X, Vol. 2. IEEE, 775-784.

[72] Junjue Wang, Brandon Amos, Anupam Das, Padmanabhan Pillai, Norman Sadeh, and Mahadev Satyanarayanan. 2018. Enabling live video analytics with a scalable and privacy-aware framework. ACM Transactions on Multimedia Computing, Communications, and Applications 14, 3s (2018), 64:1-64:24.

[73] Yilun Wang and Michal Kosinski. 2018. Deep neural networks are more accurate than humans at detecting sexual orientation from facial images. Journal of Personality and Social Psychology 114, 2 (2018), 246-257.

[74] Rolf H. Weber. 2010. Internet of Things-New security and privacy challenges. Computer Law \& Security Review 26, 1 (2010), 23-30.

[75] Thomas Winkler and Bernhard Rinner. 2012. User-centric privacy awareness in video surveillance. Multimedia Systems 18, 2 (2012), 99-121.

[76] Anthony D. Wood, John A. Stankovic, Gilles Virone, Leo Selavo, Zhimin He, Qiuhua Cao, Thao Doan, Yafeng Wu, Lei Fang, and Radu Stoleru. 2008. Context-aware wireless sensor networks for assisted living and residential monitoring. IEEE Network 22, 4 (2008), 26-33. 
[77] Jierui Xie, Bart Knijnenburg, and Hongxia Jin. 2014. Location sharing preference: Analysis and personalized recommendation. In Proceedings of the 19th International Conference on Intelligent User Interfaces (IUI'14). ACM, New York, NY, 189-198.

[78] Longqi Yang, Kevin Ting, and Mani B. Srivastava. 2014. Inferring occupancy from opportunistically available sensor data. In Proceedings of the IEEE International Conference on Pervasive Computing and Communications (PerCom'14). IEEE, 60-68.

[79] Yuchen Zhao, Juan Ye, and Tristan Henderson. 2014. Privacy-aware location privacy preference recommendations. In Proceedings of the 11th International Conference on Mobile and Ubiquitous Systems: Computing, Networking and Services. ICST (Institute for Computer Sciences, Social-Informatics and Telecommunications Engineering), Brussels, Belgium, 120-129.

[80] Jan Henrik Ziegeldorf, Oscar Garcia Morchon, and Klaus Wehrle. 2014. Privacy in the Internet of Things: Threats and challenges. Security and Communication Networks 7, 12 (2014), 2728-2742.

Received December 2018; revised July 2019; accepted September 2019 